\title{
Ion velocity distributions within the $L L B L$ and their possible implication to multiple reconnections
}

\author{
O. L. Vaisberg ${ }^{1}$, L. A. Avanov ${ }^{1,2}$, T. E. Moore ${ }^{3}$, and V. N. Smirnov ${ }^{1}$ \\ ${ }^{1}$ Space Research Institute, Moscow, Russia \\ ${ }^{2}$ NSSTC/MSFC, Huntsville, AL, USA \\ ${ }^{3}$ Goddard Space Flight Center, Greenbelt, MD, USA
}

Received: 12 November 2002 - Revised: 28 May 2003 - Accepted: 9 June 2003 - Published: 1 January 2004

\begin{abstract}
We analyze two LLBL crossings made by the Interball-Tail satellite under a southward or variable magnetosheath magnetic field: one crossing on the flank of the magnetosphere, and another one closer to the subsolar point. Three different types of ion velocity distributions within the LLBL are observed: (a) D-shaped distributions, (b) ion velocity distributions consisting of two counterstreaming components of magnetosheath-type, and (c) distributions with three components, one of which has nearly zero parallel velocity and two counter-streaming components. Only the (a) type fits to the single magnetic flux tube formed by reconnection between the magnetospheric and magnetosheath magnetic fields. We argue that two counterstreaming magnetosheath-like ion components observed by Interball within the LLBL cannot be explained by the reflection of the ions from the magnetic mirror deeper within the magnetosphere. Types (b) and (c) ion velocity distributions would form within spiral magnetic flux tubes consisting of a mixture of alternating segments originating from the magnetosheath and from magnetospheric plasma. The shapes of ion velocity distributions and their evolution with decreasing number density in the LLBL indicate that a significant part of the LLBL is located on magnetic field lines of long spiral flux tube islands at the magnetopause, as has been proposed and found to occur in magnetopause simulations. We consider these observations as evidence for multiple reconnection $X$ lines between magnetosheath and magnetospheric flux tubes.
\end{abstract}

Key words. Magnetospheric physics (magnetopause, cusp and boundary layers; solar wind-magnetosphere interactions)

\section{Introduction}

Reconnection of the interplanetary and magnetospheric magnetic fields was proposed by Dungey (1961) and is con-

Correspondence to: O. L. Vaisberg (olegv@iki.rssi.ru) sidered as a main mechanism of solar wind-magnetosphere coupling. The southward interplanetary magnetic field (IMF) may reconnect with northward dayside magnetospheric fields, forming open magnetic flux tubes at the $X$ line (Petcheck, 1964). The open magnetic tubes are identified by their rotational discontinuity properties (Sonnerup et al., 1981; Paschmann et al., 1986). Magnetosheath plasma can enter the magnetosphere along open field lines, forming the magnetospheric boundary layer. Velocity differences between the magnetosheath plasma and a rotational discontinuity lead to a velocity cutoff for the ions entering the magnetosphere and the resulting D-shaped velocity distributions on the magnetospheric field lines (Cowley, 1982). Simultaneous observations of D-shaped distributions and an open magnetopause confirm this reconnection scenario (Phan et al., 2001).

No direct evidence for multiple reconnections has previously been reported. Since the first observations of the low latitude boundary layer (LLBL) (Eastman et al., 1976), its properties and origin have been extensively studied due to its importance as a manifestation of solar wind magnetosphere coupling. It has been shown (Sckopke et al., 1981) that the LLBL is quite homogeneous, and its variability is explained by traveling vortices. Sckopke et al. (1981) divided the LLBL into the core and mantle. The case studied by Sckopke et al. (1981) was for southward IMF. Sibeck et al. (1992) suggested that the variability of the LLBL is associated with magnetopause motion. Sibeck et al. (2000) have shown that no negative density gradients are observed within the the magnetopause boundary layer and thus, the boundary layer is attached to the magnetopause. It has been shown (Hapgood and Bryiant, 1990) that all irregularities observed in the LLBL, including flux transfer events (FTEs) (Russell and Elphic, 1978), are well organized by the transition parameter (derivative of $N-T$ relationship), suggesting that magnetopause-magnetosheath motion leads to observed variability of well-organized LLBL structure.

We have previously shown the possibility to separate the LLBL into two types by its variability (Vaisberg et al., 2001). 
The highly structured LLBL is associated with either southward $B_{z}$ or variable IMF. This type of LLBL consists predominantly of the plasma transients moving with velocities close to those observed in the magnetosheath. Vaisberg et al. (1998) studied isolated LLBL events observed under southward IMF conditions and suggested that LLBL plasma transients are magnetically separated from the magnetosheath and introduced the concept of Disconnected Magnetosheath Transfer Events (DMTEs).

In this paper we analyze two cases of observations of the highly structured LLBL: one at the dusk flank of the magnetopause and another one closer to the dayside magnetopause. The longer duration of LLBL structures at the flank magnetopause allows for a more detailed analysis, while the data from short duration LLBL structures at the dayside magnetopause are used to verify the properties of highly structured LLBL. In Sect. 2 we give a short description of the observations. In Sect. 3 we describe the structure of the LLBL observed on 15 February 1996 at the flank magnetopause and analyze the ion velocity. In Sect. 4 we describe the structure of the LLBL and analyze the ion velocity distributions observed on 16 April 1996 closer to the subpolar point. In Sect. 5 we discuss the results of observations and propose their possible explanation in terms of the formation of closed spiral field structures by multiple reconnections. Section 6 is the conclusion.

\section{Observations}

The Interball-Tail spacecraft was launched on 3 August 1995 and entered the atmosphere on 13 October 2000. The Interball-Tail spacecraft was in a highly elliptic orbit with apogee $\sim 200000 \mathrm{~km}$, a period of revolution around the Earth of $\sim 4$ days and the plane of the orbit nearly perpendicular to the ecliptic plane. In 1995 and 1996 the spacecraft crossed the low-latitude magnetopause on its inbound trajectory. Interball-Tail was a spin-stabilized spacecraft with its rotation axis directed approximately towards the Sun, with a period of rotation $2 \mathrm{~min}$.

We use plasma data from the ion spectrometer SCA-1 (Vaisberg et al., 1995), which provides 3-D distributions in the range $0.05-5.0 \mathrm{keV} / \mathrm{Q}$ within $\sim 10 \mathrm{~s}$. The ion spectrometer SCA-1 has full 3-D capabilities. Its two identical sensor heads, EU-1/1 and EU-1/2, cover both hemispheres. Each sensor head consists of a toroidal electrostatic analyzer (ESA) followed by a channel electron multiplier with 8 -sectored anode. An electrostatic scanner in front of each electrostatic analyzer provides measurements over a nearly$2 \pi$ field of view. In the basic fast mode of operation the SCA-1 measures E/Q spectra over 15 energy steps in 64 directions: 8 equally spaced (by $45^{\circ}$ ) azimuthal directions by 8 polar angles relative to the Sun-directed satellite spin axis: $2^{\circ}, 17^{\circ}, 40^{\circ}, 65^{\circ}, 115^{\circ}, 140^{\circ}, 163^{\circ}$, and $178^{\circ}$. A narrow field of view $\left(2^{\circ}\right)$, and narrow energy pass-band $(\sim 10 \%)$ provide differential velocity space measurements in 960 points of velocity space. Measurements of complete energy-angular dis- tribution of ions are performed about every $10 \mathrm{~s}$, making the ion measurements almost independent of spacecraft rotation.

The ELECTRON spectrometer (Sauvaud et al., 1995) is a symmetrical "top hat" electrostatic analyzer, which provides a $180^{\circ} \times 6^{\circ}$ field of view in a meridional plane of the spacecraft with a uniform response. A chevron-mounted pair of MCP detectors with 8 associated anode sectors is used for electron angular imaging. The acceptance angle of each individual detector is $22.5^{\circ} \times 6^{\circ}$. Due to the spacecraft rotation, the full $4 \pi$ solid angle was scanned once per spin period of the spacecraft $(\sim 120 \mathrm{~s})$.

The magnetic field data are taken from the three-axial fluxgate magnetometers, MIF and FM3 (Klimov et al., 1995), with a sampling frequency up to $16 \mathrm{~Hz}$. We use the data averaged to a temporal resolution of $1 \mathrm{~s}$.

We analyze the data from two magnetopause/LLBL crossings: the 15 February 1996 magnetopause/LLBL crossing provided much more detailed data on LLBL structures when IMF was variable and the magnetosheath magnetic field was southward-duskward, as observed on Interball and Geotail spacecraft. We also analyze the data of the 16 April 1996 magnetopause/LLBL crossing at the dayside under southward IMF conditions, as observed by WIND spacecraft located close to the magnetosphere. The data of 16 April 1996 are less reliable due to a high variability of the LLBL, but they provide a crosscheck of data obtained on 15 February 1996.

\section{Characteristics of observed LLBL on 15 February 1996}

\subsection{Plasma flow parameters within the LLBL}

On 15 February 1996, the Interball-Tail spacecraft crossed the magnetopause on its inbound trajectory at $\sim 22: 50 \mathrm{UT}$. GSE coordinates of this crossing are: $X_{\mathrm{GSE}}=-3.58 R_{E}$, $Y_{\mathrm{GSE}}=17.30 R_{E}, Z_{\mathrm{GSE}}=-2.34 R_{E}, \mathrm{MLT}=18: 30$ and GM latitude $=24.9^{\circ}$. Geotail spacecraft was in the solar wind until 22:36 UT, when it crossed the bowshock and was in the magnetosheath thereafter. The measurements by Geotail indicate that a clock angle of the magnetic field varied in the range from $100^{\circ}$ and $120^{\circ}$ when Interball crossed the magnetopause and the LLBL(see Fig. 1). The Geotail spacecraft was located quite close, $\sim 5 R_{E}$ upstream of the Interball spacecraft, yielding a time lag of $\sim 1.5$ min between Geotail and Interball .

Magnetosheath plasma parameters observed by Interball were: number density $N \sim 12 \mathrm{~cm}^{-3}$, and velocity magnitude $V=\sim 220 \mathrm{~km} / \mathrm{s}$. The Alfvén velocity in the magnetosheath was $\sim 110 \mathrm{~km} / \mathrm{s}$. The magnetic field in the magnetosheath had a strong southward component. The crossing of the magnetopause occurred under large local magnetic shear, with the angle between the magnetosheath field and magnetospheric field being $\sim 140^{\circ}$. Geotail magnetic field measurements indicate that the IMF had a significant southward 
GEOTAIL, 15 Feb. 1996

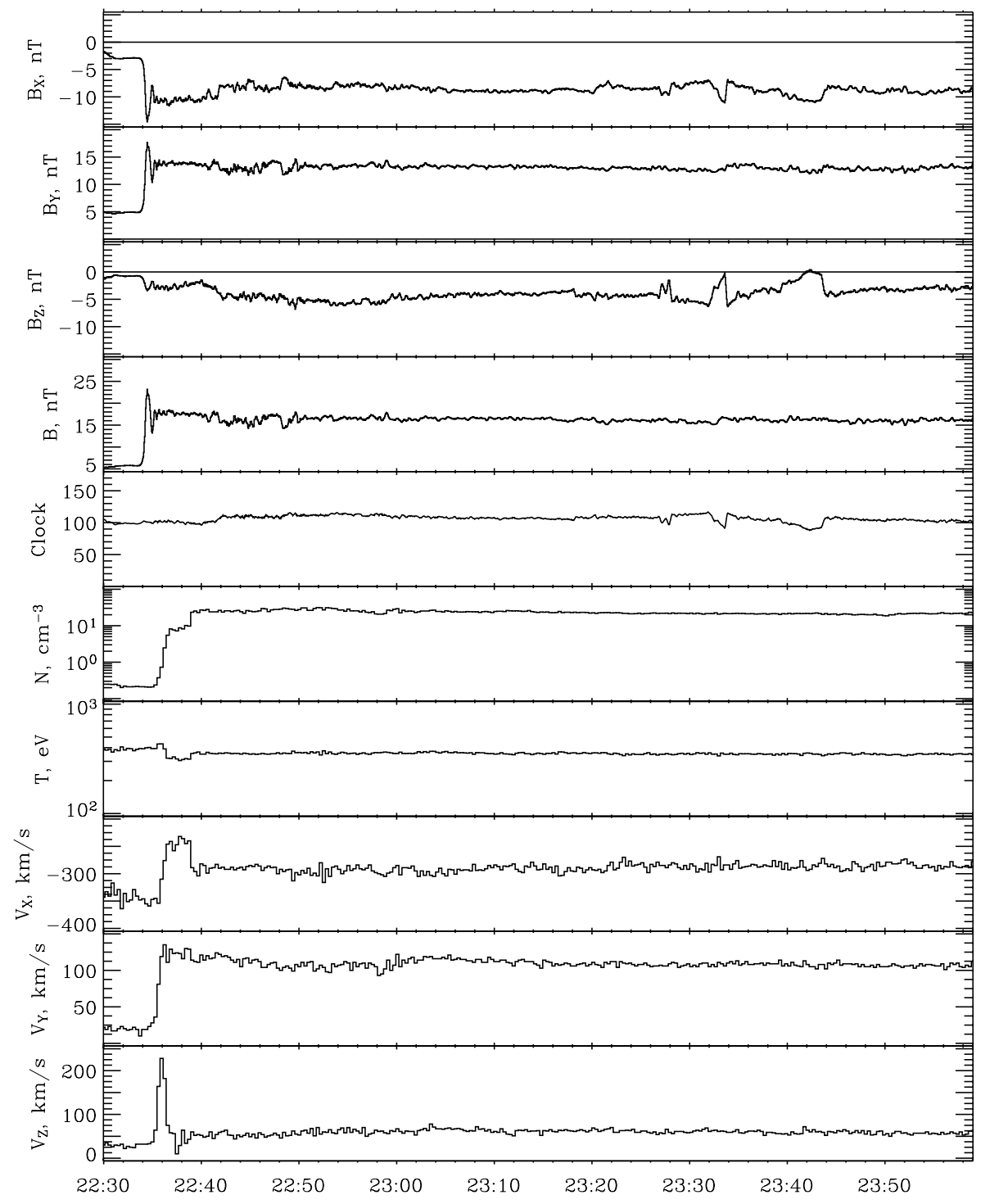

Fig. 1. Geotail magnetic field and plasma data are plotted for Interball observations of the LLBL on 15 February 1996 . From top to bottom are shown: three components of the magnetic field (GSM), magnitude of the magnetic field, clock angle defined as $\tan ^{1}\left(B_{Y G S M} / B_{Z G S M}\right)$, number density, temperature and three velocity components (GSM).

component while the Interball crossed the magnetopause and the LLBL.

Strong shear between magnetosheath and magnetospheric magnetic fields are favorable for reconnection. There are some signatures in the magnetosheath, indicating that reconnection is going on: magnetospheric ion leakage events (seen as the bursts at higher energies in the anti-sunward-looking analyzer) accompanied by stronger velocity and magnetic field variations (see Vaisberg et al., 1998, 2001).

Figure 2 shows LLBL structures observed by Interball after the magnetopause crossing. From top to bottom are shown: two energy-time spectrograms from the SCA-1 ion spectrometer, one from the sunward-looking analyzer, and another one from the anti-sunward looking analyzer, ion number density $N$, ion temperature, total velocity (in red) and $-V_{x}$-component (in black), $V_{\text {YGSM }}$ component, $V_{\mathrm{ZGSM}}$ component, Alfvén Mach number, ion beta, magnetic field magnitude (in red) and $B_{x}$ component (in black), $B_{Y G S M}$ component, and $B_{\mathrm{ZGSM}}$ component. Ion flow parameters were calculated as the moments of 10-s resolution and 3$\mathrm{D}$ ion velocity distribution measurements, with the assumption that all ions are protons. The two energy-time spectrograms are sums of the ion counting rate spectra measured along a $17^{\circ}$ cone and a $163^{\circ}$ cone relative to solar direction, respectively. Different regions near the magnetopause are indicated: (1) - magnetosheath, (2) - magnetopause current layer, (3) - boundary layer region LLBL-1, (4) - quasi-magnetosheath region and (5) - boundary layer region LLBL-2.

Regions (3), (4), and (5) in Fig. 2 have quite different 


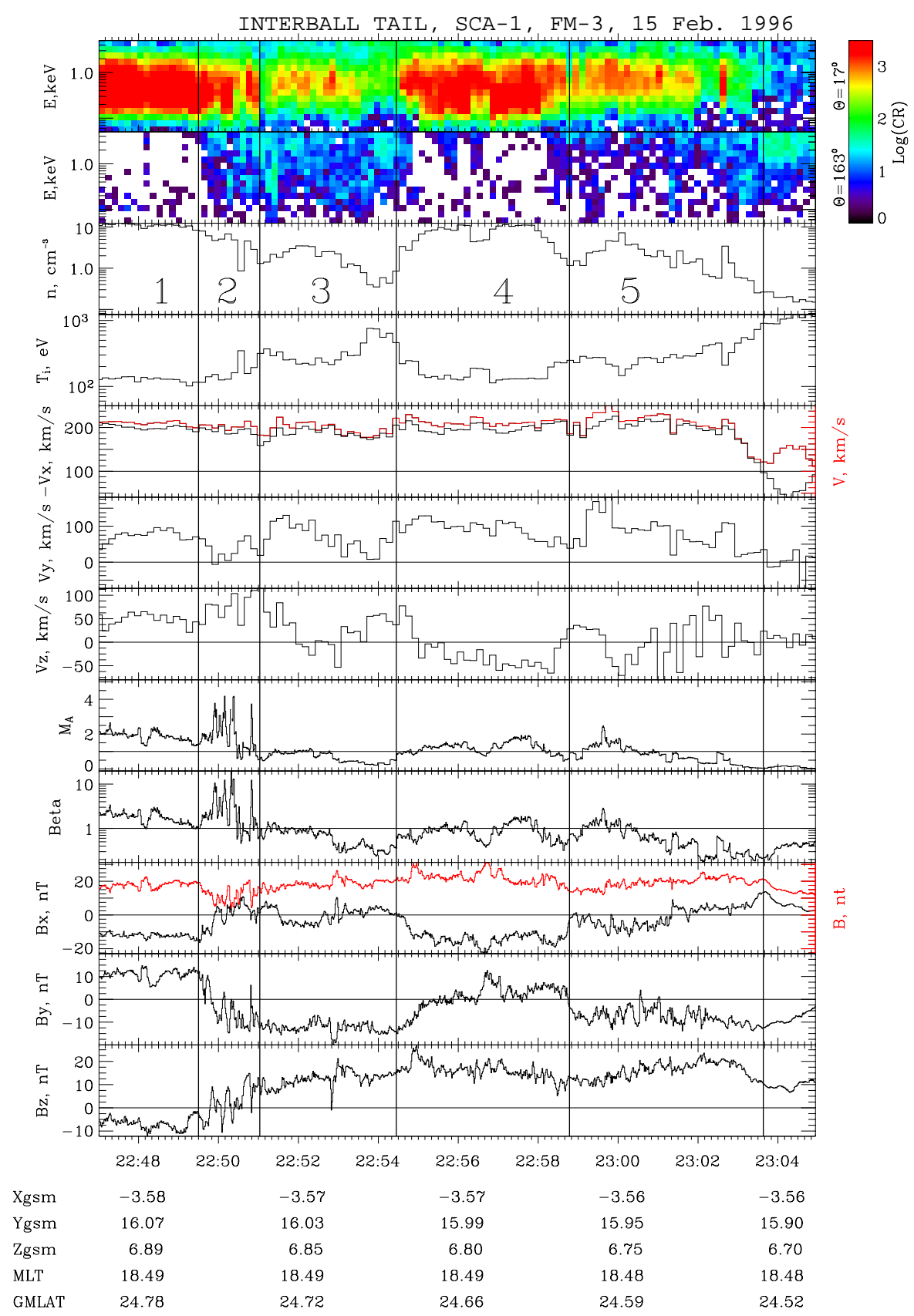

Fig. 2. Magnetopause/LLBL crossing as observed by Interball on 15 February 1996. From top to bottom are shown: two energy-time spectrograms of SCA-1 ion spectrometer, ion flow parameters number density $N$, ion temperature, total velocity (in red) and $V_{X}$ component (in black), $V_{Y G S M}$ component, $V_{\mathrm{ZGSM}}$ component, ion Mach number, ion beta, magnetic field magnitude (in red) and $B_{x}$ component, $B_{Y G S M}$ component, and $B_{Z G S M}$ component. GSM coordinates of the spacecraft location, magnetic local time, and geomagnetic latitude are shown on the bottom. See text for explanation of different regions.

plasma and magnetic field characteristics. The boundary layer regions (3) and (5) are easily distinguished from the magnetosheath by lower number density and by increased temperature. Within the time interval 22:54:27-22:58:47 UT (\#4 in Fig. 2) the plasma is magnetosheath-like, namely, the number density, ion temperature, $V_{X}$ and $V_{Y}$ velocity components, and the $B_{x}$ and $B_{y}$ magnetic field components are approximately the same as in the magnetosheath (time interval
\#1). However, Z-components of both velocity and magnetic fields have opposite signs compared to ones observed in the magnetosheath. The $B_{z}$ component within this time interval is closer to what is observed in the nearby magnetosphere, but the $B_{x}$ and $B_{y}$ components have opposite signs to those in the magnetosphere. The magnetic field vector in this region is rotated almost exactly halfway between the magnetosheath and magnetospheric directions. Thus, this time interval bears 
15 Feb. 1996

INTERBALL - ELECTRON

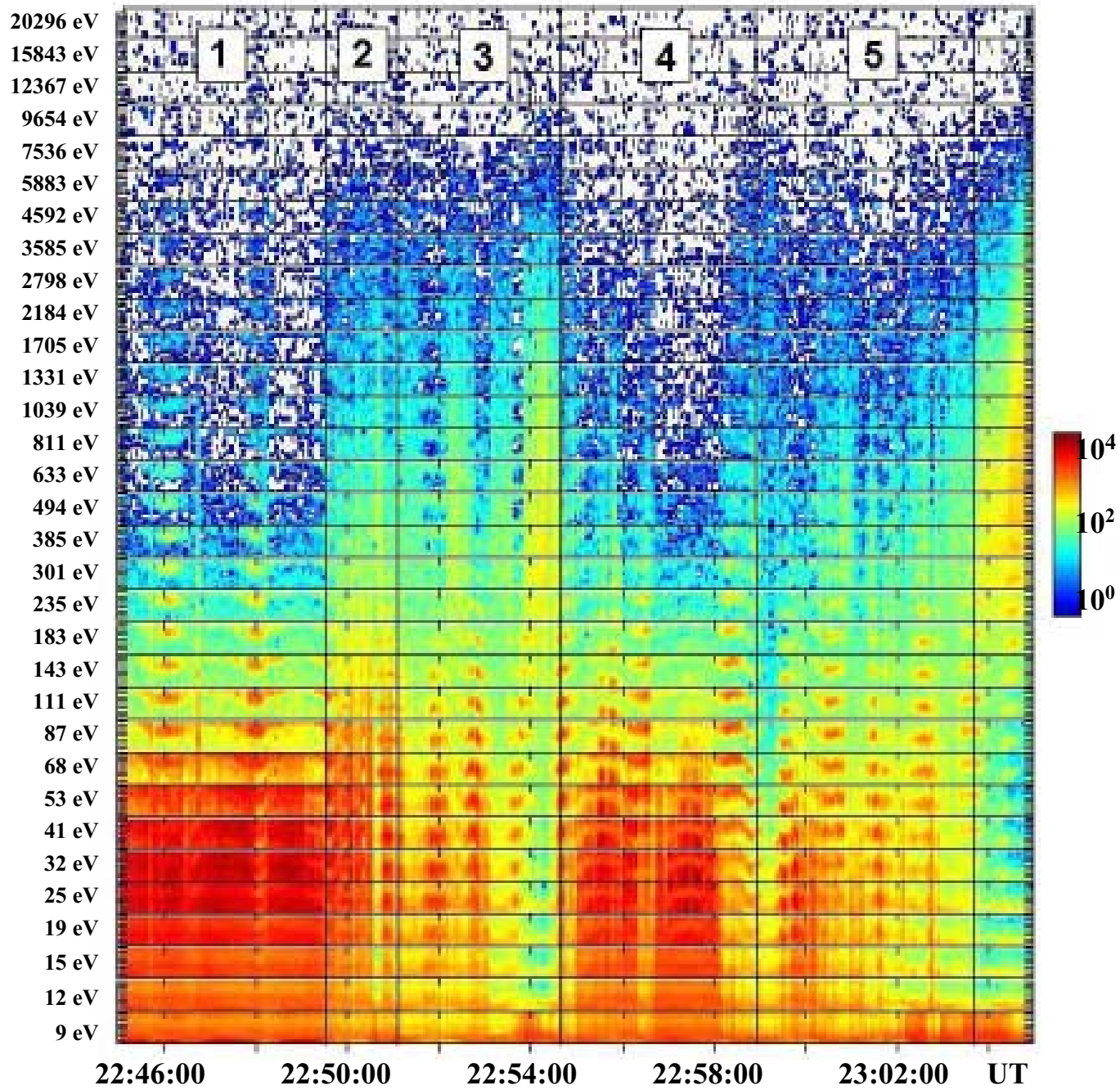

Fig. 3. Energy-time and angular spectrograms are shown for electrons in the time interval of interest on 15 February 1996. Each horizontal panel represents the counting rate of electrons with the energy indicated to the left. The distribution of the counting rate along the ordinate within each energy band shows an angular distribution of electrons in the meridional plane of the satellite, in which the Sun is on the pole. The anti-sunward moving electrons are on the upper part of each panel, and sunward moving electrons are on the lower part of the panel. Three dimensional velocity distribution of electrons is measured within a 2-minute period of the satellite's rotation.

some properties of the magnetosheath, and some properties of the magnetosphere; however, it is hardly possible to consider it as part of the magnetosphere. At the same time, the different sign of $B_{z}$ from the one of the magnetosheath (see Geotail measurements of Fig. 1), and, specifically, the lower Alfvén Mach number $M_{A}$ compared to the magnetosheath makes it difficult to consider it as part of magnetosheath flow. The origin of this magnetosheath-like structure needs further explanation.

Figure 3 shows the combined energy-time and angular distributions of electrons for plasma regions shown in Fig. 2. Each horizontal panel represents the counting rate of electrons with the energy indicated to the left. The distribution of the counting rate along the ordinate shows an angular distribution of electrons in the meridional plane of the satellite, so that the anti-sunward moving electrons are shown on the upper part of each panel, and sunward moving electrons are shown on the lower part of the panel. As the satellite rotates along the nearly sunward oriented axis with a 2-min period, the ELECTRON spectrometer samples 3-D velocity distribution of electrons. As a result, an omni-directional distribution will be seen as a non-modulated color band, a trapped distribution will be seen as a bright sinusoidal band, a monodirectional field-aligned beam will be seen as a maximum once per 2-min rotation, and a bi-directional field-aligned distribution will be seen as 2 maxima per 2-min rotation (periodicity of $1 \mathrm{~min}$ ).

The regions in this figure are marked according to those in Fig. 2. There is a leakage of electrons with energies up to $\sim 5 \mathrm{keV}$, indicating connection of the magnetosheath (region 


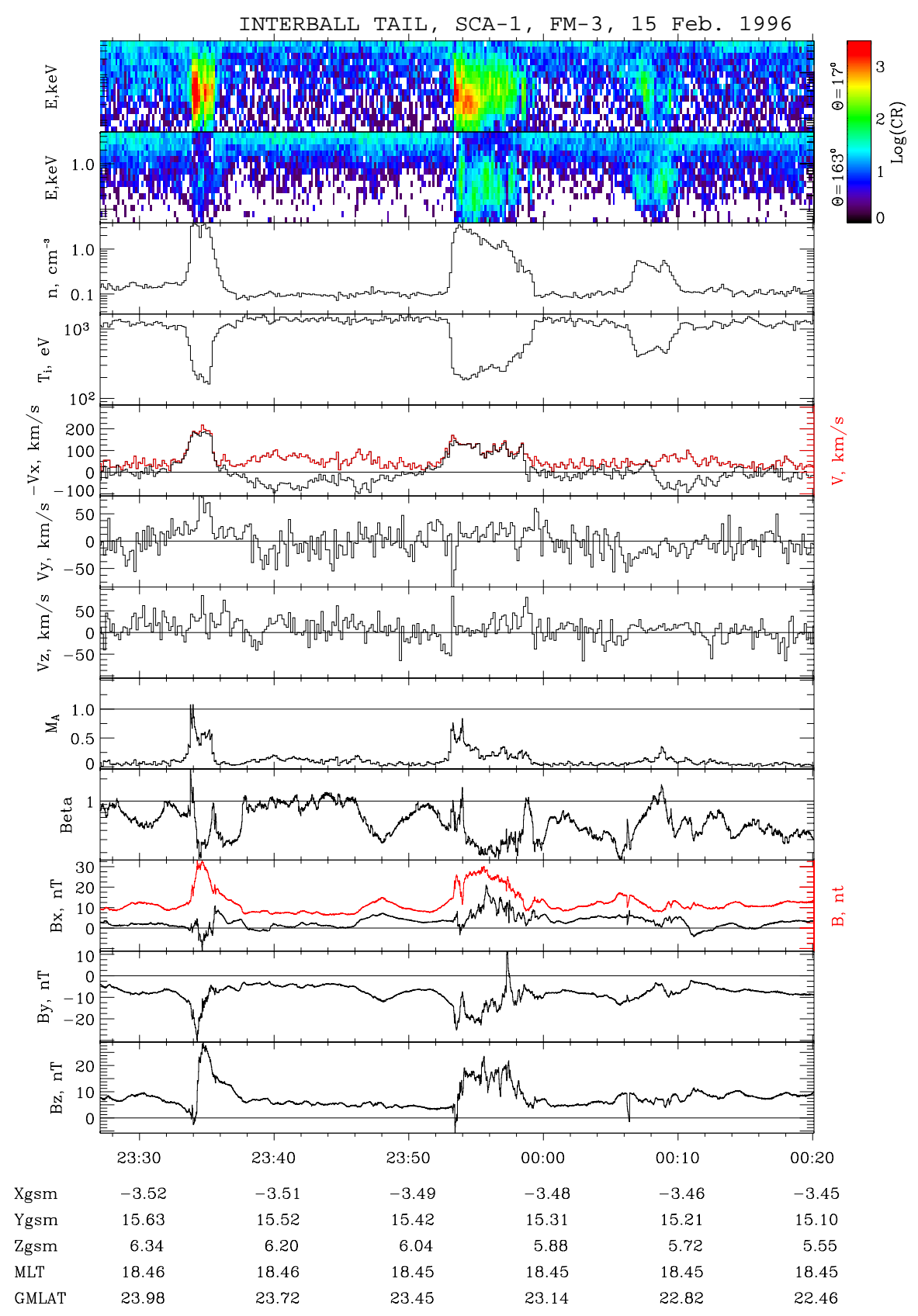

Fig. 4. Three LLBL transients are plotted as observed by Interball on 15 February 1996. See Fig. 2 caption for explanation. Note that within this succession of transients there is an inverse relationship between number density and velocity, on one side, and ion temperature, on the other side.

1) to the magnetopause. The magnetopause current layer (region 2) is characterized by sporadic bursts of electrons that, on average, are more energetic than magnetosheath electrons. The electrons show an increase in the parallel temperature in the boundary layer regions 3 and 5, as seen from 1-min periodicity of the electron flux maxima. These bi-directional electrons are specific to the boundary layer (Thomsen et al., 1987). The electron distributions in the magnetosheath-type region 4 are similar to those observed in the magnetosheath proper but several differences can be pointed out. The high energy part of the magnetosheath distributions (region 1, energies $68-183 \mathrm{eV}$ ), is streaming along the magnetic field in one direction because it is encountered once per spacecraft spin. On the other hand, the distribution in region 4 exhibits two maxima per spin (most clearly seen at 41 and $53 \mathrm{eV}$ ) indicating counter-streaming electrons usually observed in the boundary layer.

Figure 4 shows three isolated magnetosheath-type plasma 
a)

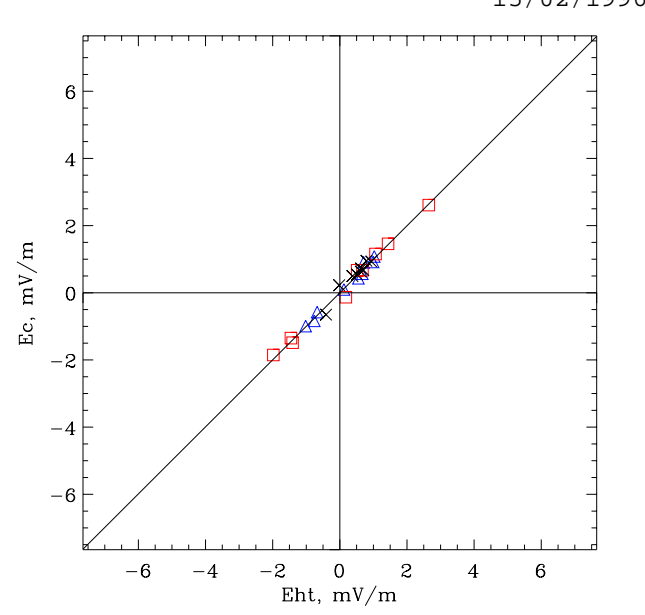

$\operatorname{Vht} 0(\mathrm{x}, \mathrm{y}, \mathrm{z})=[-264.9,146.6,-67.0], \mathrm{km} / \mathrm{s}$ Aht $=[1.993,-1.757,2.944], \mathrm{km} / \mathrm{s}^{2}$ $\operatorname{Vht}(\mathrm{x}, \mathrm{y}, \mathrm{z})=\left[\begin{array}{lll}-190.5,96.0, & 7.6\end{array}\right], \mathrm{km} / \mathrm{s}$

$\mathrm{K}=0.996+/-0.025 ; \mathrm{R}=0.992 ; \mathrm{D} / \mathrm{D} 0=0.015$

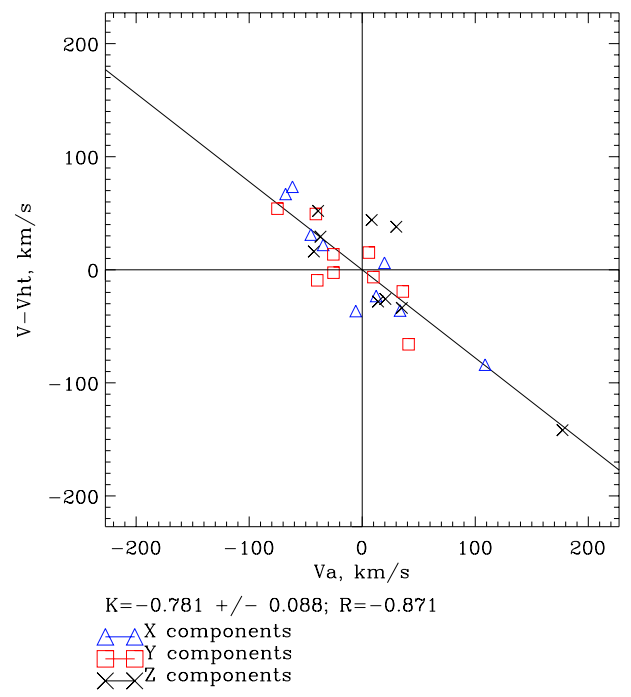

b)

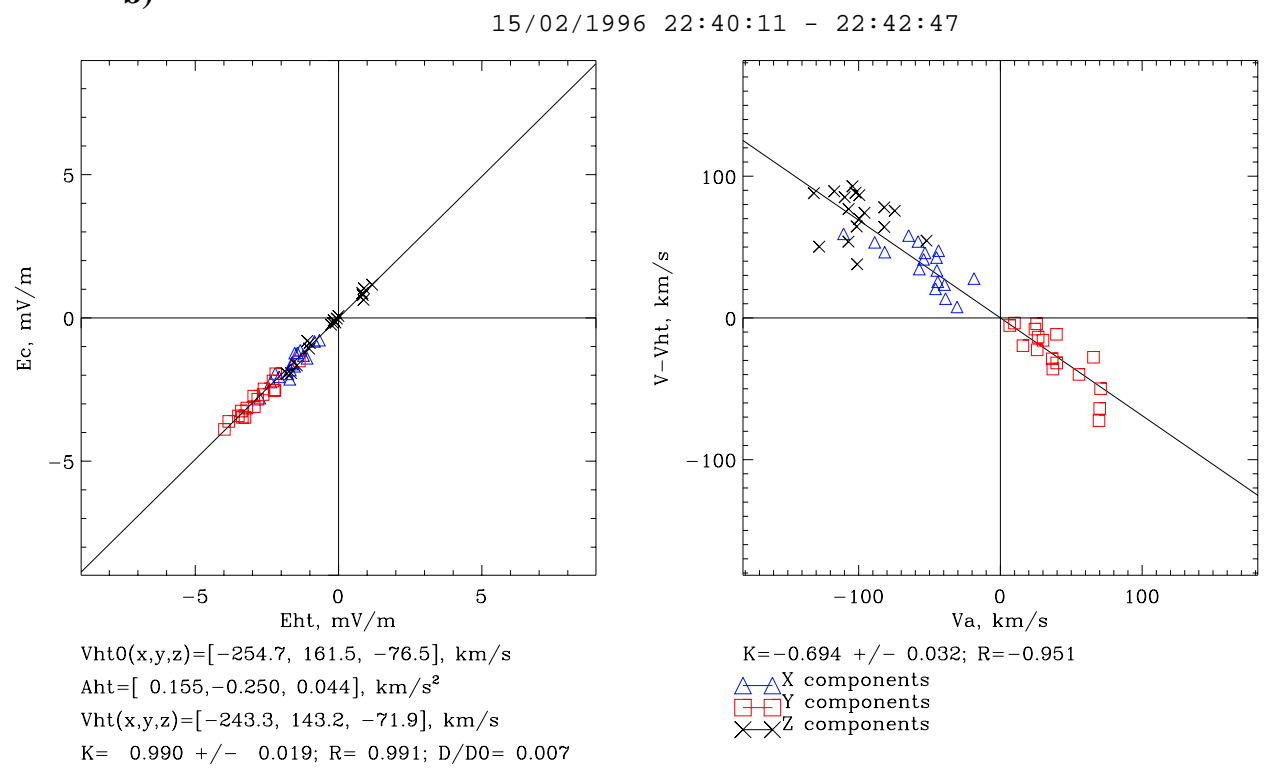

Fig. 5. Scatter plots (a) is for magnetopause crossing and (b) is for the magnetosheath region adjacent to the magnetopause. (Left) A component-by-component comparison is made of the convective electric field $\boldsymbol{E}_{\boldsymbol{c}}=-\boldsymbol{V} \times \boldsymbol{B}$ versus electric field $\boldsymbol{E}_{\boldsymbol{H} T}=-\boldsymbol{V}_{\boldsymbol{H}} \boldsymbol{T} \times \boldsymbol{B}$. The defined components of the HT and HT frame speed and acceleration in GSE are given. $K$ and $R$ are a slope and correlation coefficient between the convective electric field and $\boldsymbol{E}_{\boldsymbol{H} \boldsymbol{T}}$, respectively; (Right) Result of tangential stress balance referred to as Walen relations. $K$ is a slope and $R$ is correlation coefficient between the flow velocity in the HT frame and the corresponding Alfvén velocity. Triangles, open boxes and crosses correspond to $X, Y$ and $Z$ components, respectively.

transients observed $\sim 45$ min after the magnetopause crossing. The format of Fig. 4 is the same as for Fig. 2. These transients display a progressive (from left to right) decrease in velocity and density and increase of temperature from values compared to those observed in the magnetosheath. Some of these transients have the magnetic field signature of FTEs. Two of three plasma transients are moving, while the third one is nearly stationary relative to surrounding plasma. Anal- ysis of three LLBL transients was made in (Vaisberg et al., 1998). It was shown that they have a non-symmetric structure with a faster and denser leading part and a turbulent trailing part. These transients keep nearly the same structure while their properties change in a systematic way with their density diminishing from the first to the last one. The plasma and magnetic structure of these LLBL transients and their evolution with the distance from magnetopause suggest that 


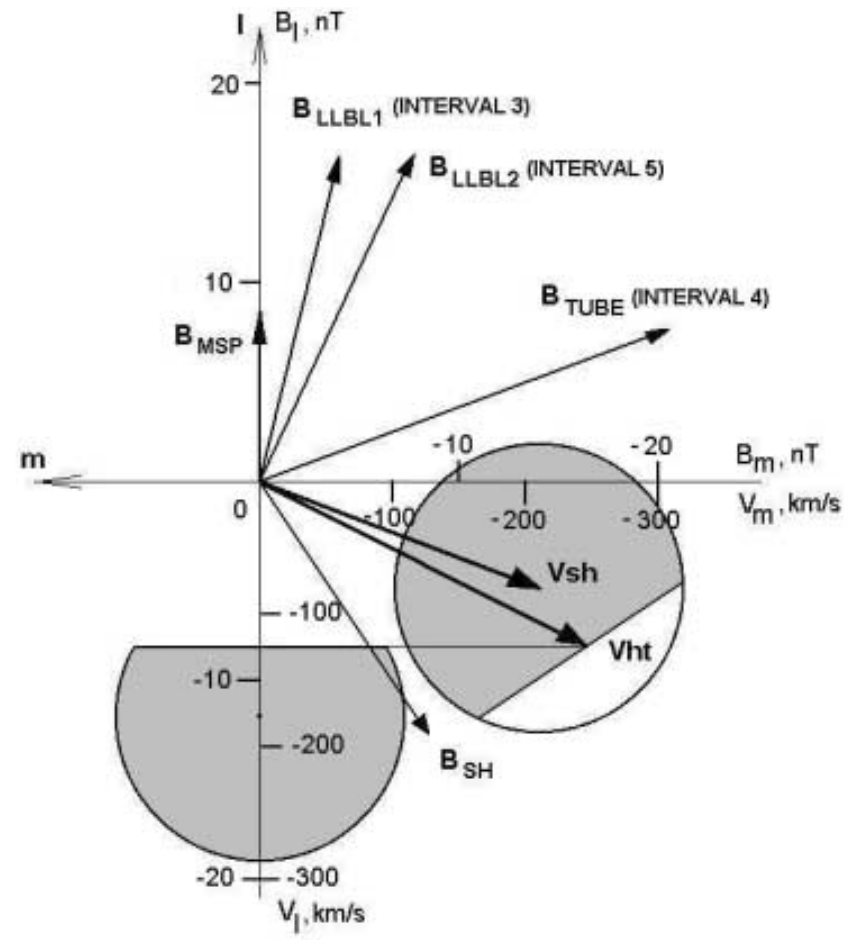

Fig. 6. A Cowley (1982) diagram is shown for 15 February 1996. It shows projections of the velocities and magnetic field vectors on the magnetopause plane determined by minimum variance ( $n$ is pointing out of the page, $l$ is along the magnetospheric magnetic field, and $m$ completes the right-hand side coordinate system). $V_{s h}$ is the magnetosheath plasma velocity and $V_{H T}$ is the de HoffmanTeller frame velocity. $B_{s h}$ and $B_{m s p}$ are magnetic field in the magnetosphere and in the magnetosheath, respectively. Average magnetic fields vectors for LLBL intervals 3, 4, and 5 in Fig. 2 are also shown. The magnetosheath ion velocity distribution is shown schematically by a circle centered on the magnetosheath velocity vector. A straight line drawn through the end of the dHT velocity vector perpendicular to the $B_{s h}$ vector determines the velocity space (gray-filled) area from where magnetosheath ions can enter magnetospheric field lines reconnected to the magnetosheath magnetic field to the north of the spacecraft. The ion velocity distribution that should be observed on the open magnetospheric field line is shown schematically by a gray-filled area moving opposite to the magnetospheric magnetic field direction with a velocity cutoff placed at the projection of the HT velocity on the magnetospheric magnetic field direction.

they are not magnetically connected to the magnetosheath. They were dubbed in (Vaisberg et al., 1998) as Disconnected Magnetosheath Transfer Events, or DMTEs, associated with (non-stationary) reconnection.

\subsection{Reconnection geometry on 15 February 1996}

The properties of the magnetopause indicate that reconnection takes place during this time interval. The magnetopause current layer (2) is defined by the change of sign in the three magnetic field components, by depression of the magnetic field magnitude, and by an increased ion beta. A good (with correlation coefficient 0.995) de Hoffman-Teller frame (HT frame) and a good Walen relation (with a negative slope of the fit between measured and calculated Alfvén velocity of $\sim 0.76$ ) were found for the time interval 22:49:1422:50:32 UT, according to method of Sonnerup et al. (1987) (Fig. 5a). The HT frame has been found as the reference frame in which the mean square of the convective electric field, $D=\left\langle\left|\left(\boldsymbol{V}-\boldsymbol{V}_{\boldsymbol{H} T}\right) \times \boldsymbol{B}\right|^{2}\right\rangle$ has a minimum (Sonnerup et al., 1987). The ratio of $D / D 0$ (where $D 0=\left\langle|\boldsymbol{V} \times \boldsymbol{B}|^{2}\right\rangle$ ) is used as a measure of the quality of the defined HT frame velocity and $D / D 0$ should be small $(\ll 1)$ (Khrabrov and Sonnerup, 1998). This indicates that the magnetopause current layer is a rotational discontinuity, and a negative slope of the Walen relation implies that the reconnection site is located northward relative to the spacecraft position. This is in agreement with the negative deviation of the $Z$ component of plasma velocity for a significant part of the LLBL, compared to that of the observed in the magnetosheath. This also agrees with the expected location of the antiparallel reconnection (Crooker, 1979; Luhmann et al., 1984) at the northdusk sector for the IMF clock angle $100^{\circ}-120^{\circ}$, as measured by Geotail. However, positive $V_{Z}$ values within part of the current layer indicate a more complex nature of this transition, possibly associated with the deviation from planar geometry. De Keyser et al. (2001) performed a very detailed analysis of this magnetopause crossing and concluded that the magnetopause was not steady. Strong acceleration of the de Hoffman-Teller frame during the time interval where Interball crossed the current layer (see numbers on Fig. 5a) also indicates that it is locally non-steady and complicated.

A good de Hoffman-Teller frame is found for the magnetosheath boundary layer adjacent to the magnetopause where plasma velocity and magnetic field variations are observed, as well as sporadic leakage of more energetic ions and electrons from the magnetosphere. The calculated de HoffmanTeller frame and Walen relation for one such interval is shown in Fig. 5b. This indicates that the magnetosheath boundary layer includes reconnected flux tubes. Low values of acceleration of the de Hoffman-Teller frame and consistent values of calculated velocities show that the reconnection process is quasi-steady. The negative slope of the Walen relation confirms that reconnected flux tubes originate to the north of the spacecraft location, in accordance with other data.

The de Hoffman-Teller frame velocity in the magnetosheath boundary layer is quite close to the de HoffmanTeller frame velocity for the beginning of the magnetopause current layer. Due to this and due to the consistency of the calculated de Hoffman-Teller frame velocities for different time intervals in the magnetosheath boundary layer, we believe that this HT frame characterizes the observed magnetopause and LLBL crossing. For analysis of velocity distributions in the LLBL we adopted the HT frame calculated for a $\sim 10$-min time interval within the magnetosheath boundary layer: $V_{H T}(\mathrm{GSE})=(-240,128,-48) \mathrm{km} / \mathrm{s}$.

Figure 6 shows the geometry of reconnection on a $\boldsymbol{l}-\boldsymbol{m}$ plane of the normal coordinate system lmn. An average 

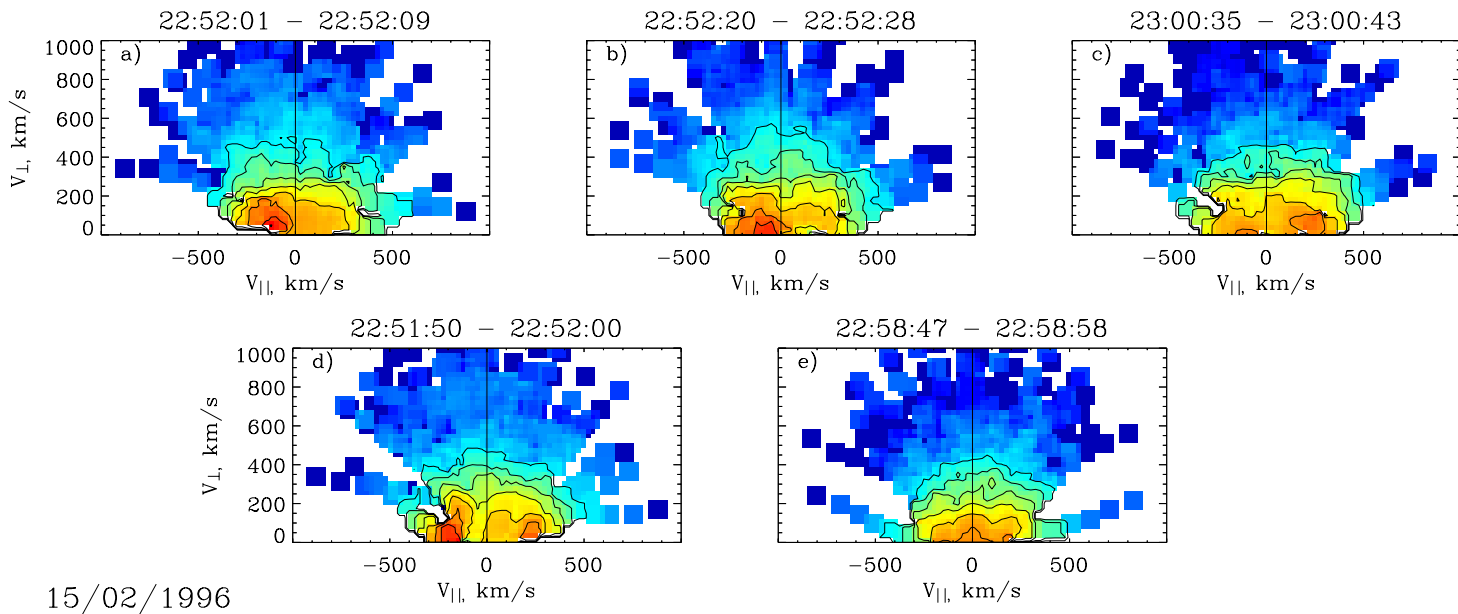

$15 / 02 / 1996$

d a, b

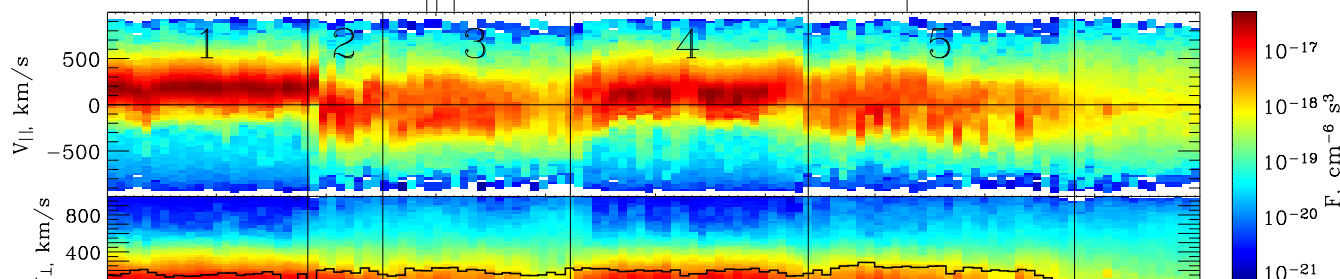

15

हु 15

\begin{tabular}{lr}
$z$ & 5 \\
\hline & 10 \\
\hline & 0
\end{tabular}

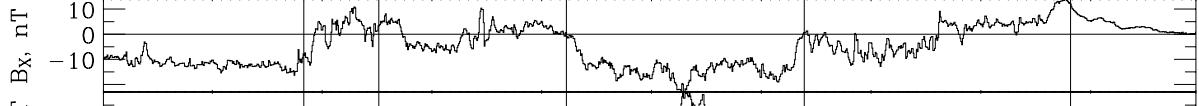

ต

芒

导 10

(a)

$\mathrm{X}_{\mathrm{GSE}}$

$Y_{G S E}$

$\mathrm{Z}_{\mathrm{GSE}}$

MLT

GMLAT $\begin{array}{rl}10 & 0 \\ -10 & 10\end{array}$

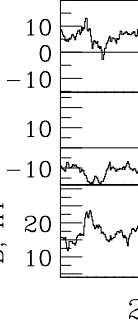

$22: 48 \quad 22: 50$

$-3.58$

17.34

$-2.30$

18.49

24.78

Min

\section{r}
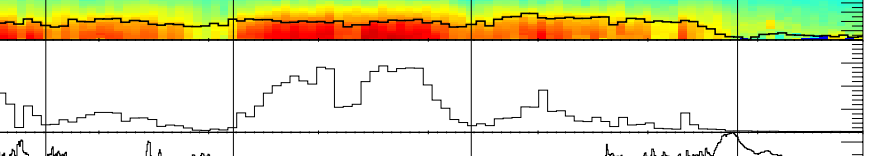

(n)

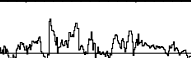

(ling in

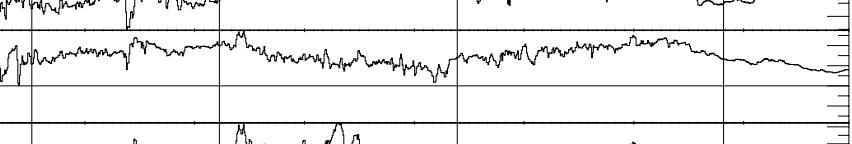

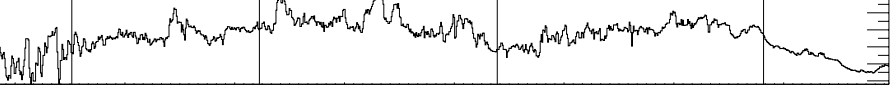

$22: 52 \quad 22: 54$

$-3.57$

17.28

$-2.33$

18.49

24.72

$22: 56$
-3.57
17.21

17.21

$-2.35$

18.49

24.66

22:58

$23: 00$
-3.56

-3.56
17.15

17.15
-2.37

18.48

24.59

23:04

$-3.56$

17.09

$-2.40$

18.48

24.52

Fig. 7. Representative ion velocity distributions are given within the LLBL near the magnetopause on 15 February, 1996 (the same time interval as in Fig. 2). Two color-coded $V_{\|}$and $V_{\perp}$ velocity-time spectrograms show the phase space density integrated over perpendicular velocity (upper spectrogram) and the phase space density integrated over parallel velocity (lower spectrogram), after the velocity component transverse to the local magnetic field direction was subtracted. This transverse plasma velocity component is superimposed on the lower spectrogram (black line). Ion number density is shown on the bottom. Lettered lines above the spectrograms show locations where respective $V_{\|}-V_{\perp}$ velocity distributions were measured. GSE coordinates of the spacecraft, magnetic local time, and geomagnetic latitude are shown on the bottom. Time interval for each spectrogram is indicated on the top. See text for a more detailed explanation.

magnetic field in the magnetosheath, three LLBL intervals in the magnetosphere are shown along with magnetosheath plasma velocity and the de Hoffmann-Teller frame velocity in projection to the magnetopause plane. The direction of the $\boldsymbol{V}_{\boldsymbol{H} T}$ vector indicates that the reconnection $X$-line is located northward and westward of the spacecraft.

The diagram in Fig. 6 can be used to estimate the properties of magnetosheath plasma that should enter the open field lines using the approach developed by Cowley (1982). A circle centered on $V_{s h}$ schematically shows the magnetosheath velocity distribution. The hatched area within this circle is part of the velocity distribution that is allowed to enter the magnetosphere along the reconnected field line. Upon entry into the magnetospheric part of the field line, these particles will obtain additional velocity equal to the projection of the de Hoffman-Teller velocity to the magnetospheric field line 

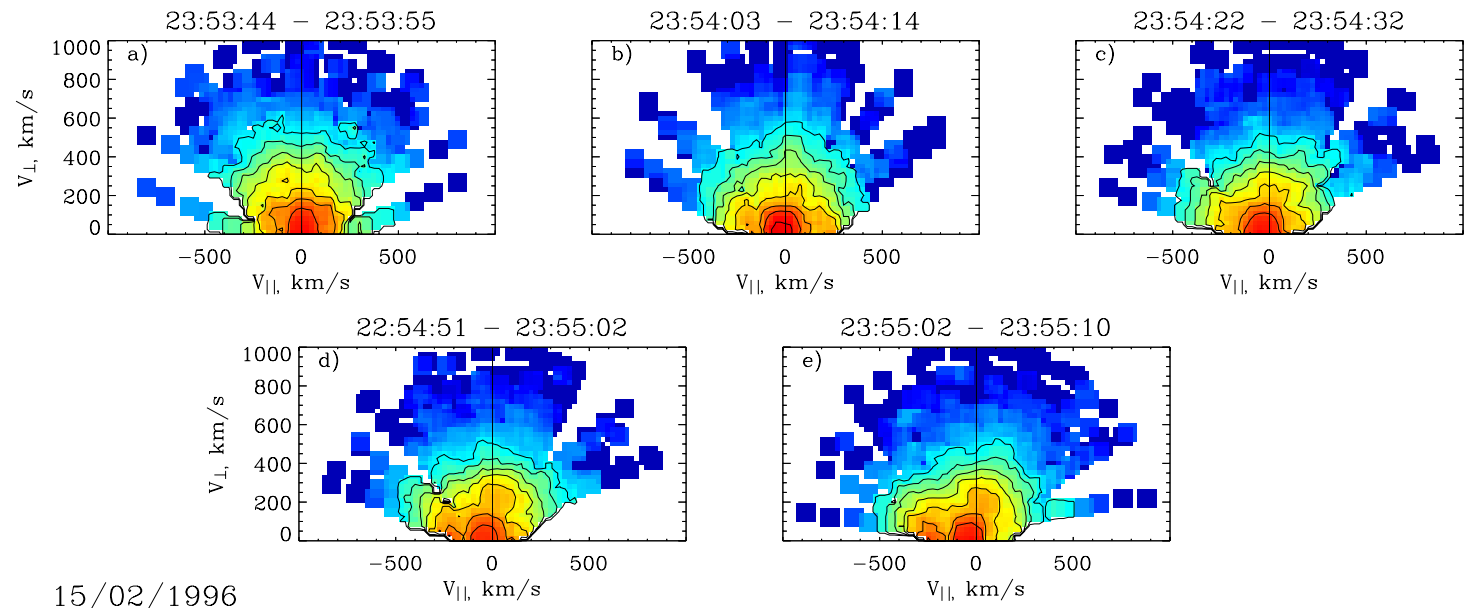

$15 / 02 / 1996$

ab c de

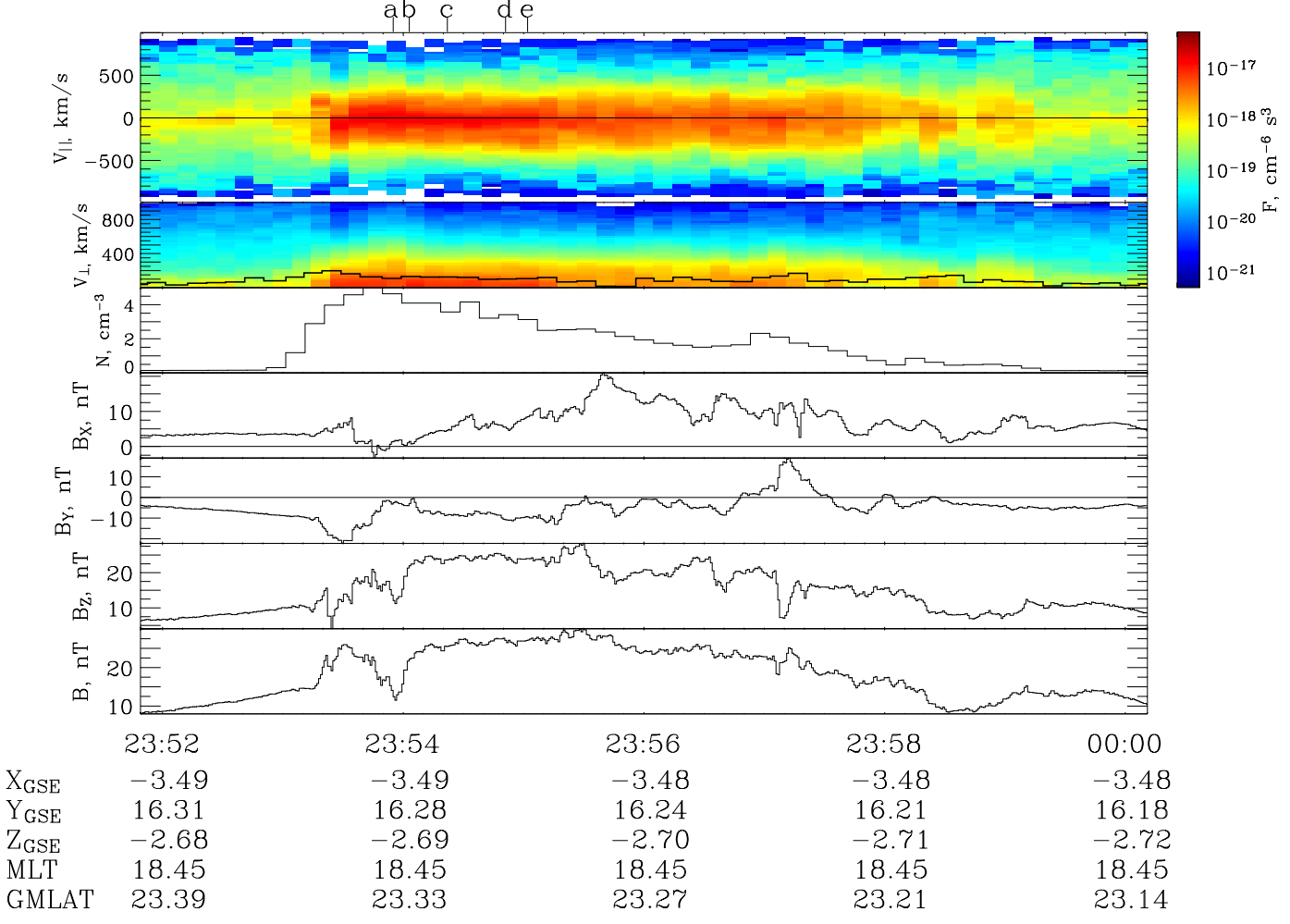

Fig. 8. Ion velocity distributions within transient observed at $\sim 23: 35$ UT on 15 February 1996. Format of the figure is the same as in Fig. 10 . Five representative ion velocity distributions (see text for explanation) are shown on the top.

(equal to $125 \mathrm{~km} / \mathrm{s}$ ). The resulting D-shaped velocity distribution is shown as a hatched area at $-\boldsymbol{l}$ axis. This gives us background information for subsequent comparison of velocity distributions in the LLBL with what is expected from reconnection for the given geometry.

Indication of the direction to the reconnection site from the calculated HT velocity frame is in reasonable agreement with the predictions of the models. For the IMF clock angle that was observed on 15 February 1996, the model of Crooker (1979) predicts the location of reconnection site where the magnetic fields are closest to being antiparallel in the northdusk sector. The model of Moore et al. (2002) predicts a location of the reconnection site where antiparallel components of two magnetic fields are maximal, being also being in the north-dusk sector.

\subsection{Ion velocity distributions observed in the LLBL on 15 February 1996}

Figure 7 shows the ion velocity distributions within the LLBL plasma observed after the magnetopause crossing. We show five representative examples of ion velocity distributions within the LLBL1 and LLBL2 (regions (3) and (5) in Fig. 2). Observed types of velocity distributions include: (a) plasma injections along the negative magnetic field direction, 
(b) a stronger component along the negative direction of $B$ and a weaker component along the positive direction of $B$, (c) a weaker component along the negative direction of $B$ and a stronger component along the positive direction of $B$, (d) two nearly identical ion components, and (e) two oppositely moving components and the third component at nearly zero velocity. Only type (a) can be expected for the field line opened northward relative to the spacecraft location.

These velocity distributions in the magnetic coordinate frame were obtained in the following manner. First, the plasma velocity vector in the spacecraft (laboratory) coordinate system was calculated with a $\sim 10$ s temporal resolution from each 3-D ion velocity distribution measured by SCA1. Magnetic field measurements were averaged for the same time intervals as for 3-D ion measurements. Then the velocity component transverse to the magnetic field direction was subtracted from each of the 960 vectors representing velocity space bins of SCA-1 in the laboratory coordinate frame. In the next step, every measurement of the phase space density for a particular 3-D measurement frame was rotated into a half-plane containing the averaged magnetic field vector. A resulting scatter plot of phase space densities was averaged over the grid of $100 \mathrm{~km} / \mathrm{s}$. Incomplete velocity space coverage by SCA-1 leads to gaps on displayd planar cross sections of velocity distributions. The result of this data processing is the velocity space distribution in a magnetic coordinate system with the origin at the vector difference between the plasma bulk velocity in the laboratory coordinate system and its component perpendicular to the local magnetic field direction.

Representative velocity distributions are shown in Fig. 7 in the context of $V_{\|}-V_{\perp}$ color-coded velocity-time spectrograms. These velocity-time spectrograms were obtained by the integration of $V_{\|}-V_{\perp}$ velocity distributions over perpendicular velocity (upper spectrogram) and over parallel velocity (lower spectrogram). Thus, these spectrograms show parallel and perpendicular velocity distribution versus time. The calculated velocity component transverse to the magnetic field direction is shown by a solid line on the second panel from the top. Ion number density, magnetic field components and magnitude are shown in the next five panels. It is seen that the boundary layer plasma is moving quite steadily transverse to the magnetic field. What seems to be important is the frequently observed significant ion density at zero velocity and at positive velocities relative to the magnetic field.

The transient observed at $\sim 23: 55$ UT had sufficient duration to allow for a more detailed analysis of the ion velocity distributions for different ion number densities within the LLBL. This transient is shown in Fig. 8 in a $V_{\|}-V_{\perp}$ magnetic coordinates frame, along with several representative velocity distributions. This transient has a clear double structure with leading fast and denser plasma, and a trailing, less dense part (see detailed analysis of this transient or DMTE in Vaisberg et al., 1998). In the leading part of this transient the density is steadily decreasing onward from the maximum near the front edge. In the trailing part, the density remains nearly constant most of the time. Weak injections anti-parallel and parallel

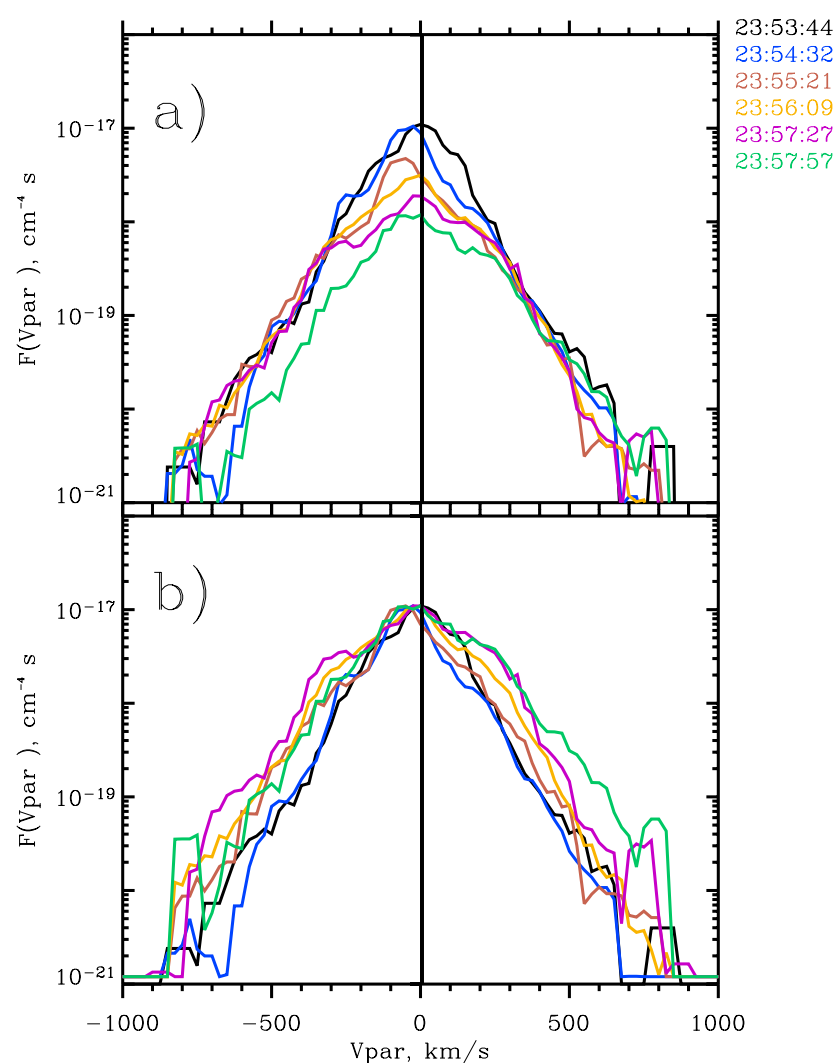

Fig. 9. Six ion velocity distributions (times of measurements are shown to the right) are given within transient at $\sim 23: 55$ UT (top). Bottom: the same velocity distributions normalized to the maximum intensities.

to the magnetic field direction are observed occasionally (e.g. velocity distribution (c). However, the main feature of the velocity distribution is a component at zero parallel velocity. It dominates in the first part of the transient and subsequently diminishes (velocity distributions (b, $d$, and e).

The long duration of this DMTE and the nearly monotonic variation of the number density in its leading part allows one to analyze how velocity distribution in the LLBL changes with the number density change. Figure 9 shows the evolution of ion velocity distributions within the leading part of this transient. We have chosen 6 ion velocity distributions with progressively decreasing number densities. In this subset of spectra (top part of Fig. 9) the flux at zero velocity is decreasing, while the flux at the wings remains nearly the same. It is easier to see how the velocity distribution evolves with decreasing number density when one normalizes the spectra to their maximum values (bottom part of Fig. 9). This normalization indicates that the relative contribution of wings in the velocity distribution is increasing as the density decreases, and that they become less steep (more heated).

We made a comparison between observed magnetosheathtype plasma injections, along the negative magnetic field direction, and theoretical expectations. Three cases of a high 

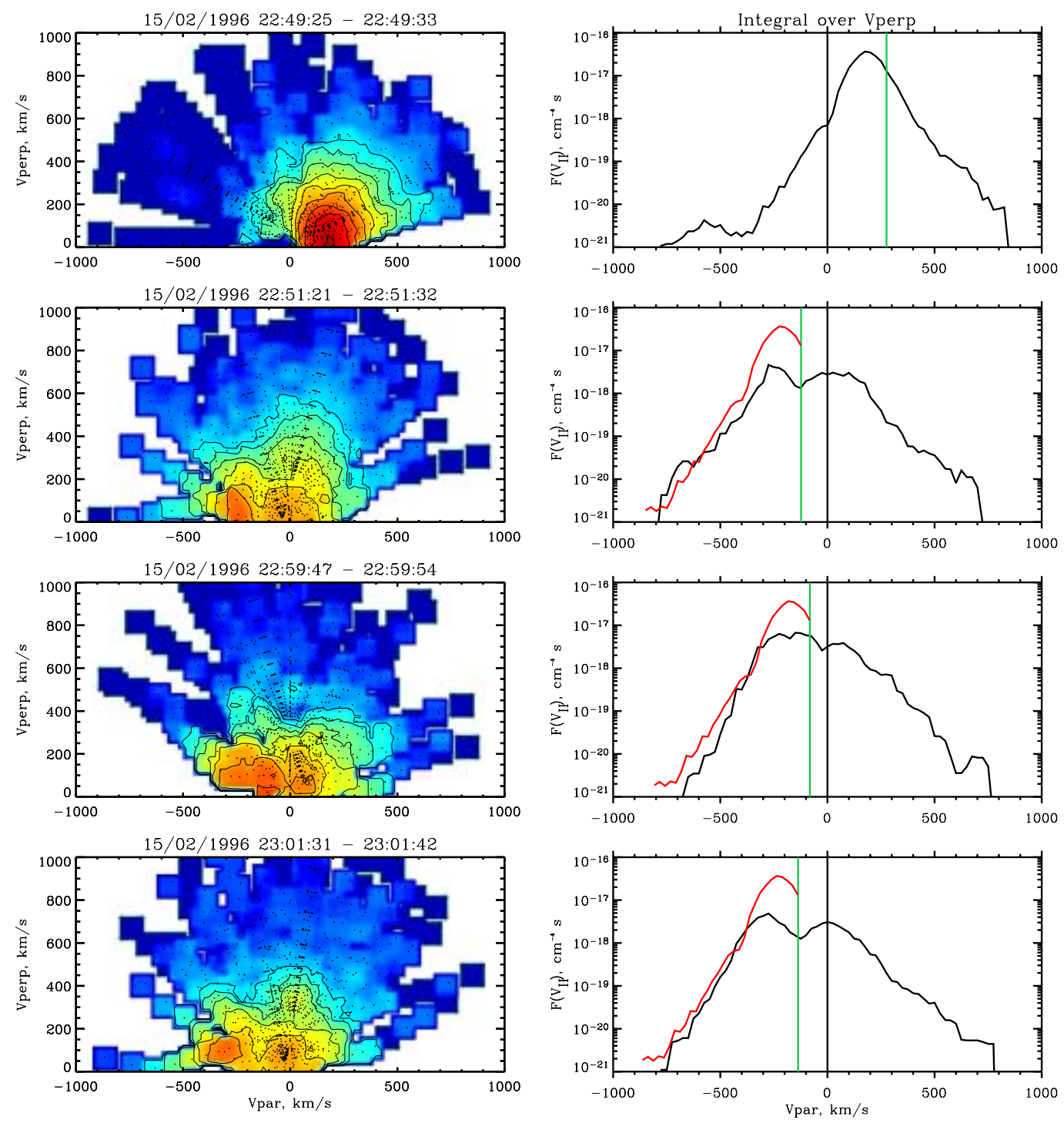

Fig. 10. Magnetosheath velocity distribution observed before magnetopause crossing on 15 February 1996 (top) and three LLBL velocity distributions with a high-density, high-speed ion component along negative magnetic field direction observed within LLBL intervals 3 and 5 in Fig. 2. $V_{\|}-V_{\perp}$ velocities space cuts are on the left, $V_{\|}$-velocity distributions are on the right. The deHoffman-Teller frame velocity cut is shown by a vertical green line on magnetosheath $V_{\|}$-velocity distribution. Red lines at each $V_{\|}$-velocity distribution in the LLBL show the magnetosheath plasma velocity distributions that should enter magnetospheric field lines if they are reconnected to magnetosheath field lines. The velocity cut location on the $V_{\|}$-coordinate was determined as a projection of dHT velocity on the local magnetic field direction for each case.

density component with high convection velocity were selected within the LLBL (time intervals 3 and 5 in Fig. 2). Figure 10 shows these cases in comparison with the magnetosheath velocity distribution observed just before the magnetopause crossing. The de Hoffman-Teller frame velocity component parallel to the magnetosheath magnetic field is shown by a green vertical line on the $V_{\|}$-distribution in the magnetosheath. Projections of this dHT velocity to average magnetic field directions for each LLBL velocity distribution is shown by green vertical line on the $V_{\|}$-distributions. According to Cowley's (1982) scheme we expect that magnetosheath particles with negative velocities relative to the green line on the $V_{\|}$-distribution will enter the open field line. These particles should be observed at velocities to the left of each green line on the $V_{\|}$-distributions (right column in Fig. 10). The red curves mark each of these velocity distributions. Expected and observed velocity components at negative velocities are quite close in the locations of maxima and in the temperature. Figure 10 suggests that high-density injections of magnetosheath plasma along negative magnetic field directions can be explained by magnetosheath plasma entry onto the open field lines reconnected to magnetosheath field lines, to the north of the spacecraft.

We also selected different cases of LLBL velocity distribu- 

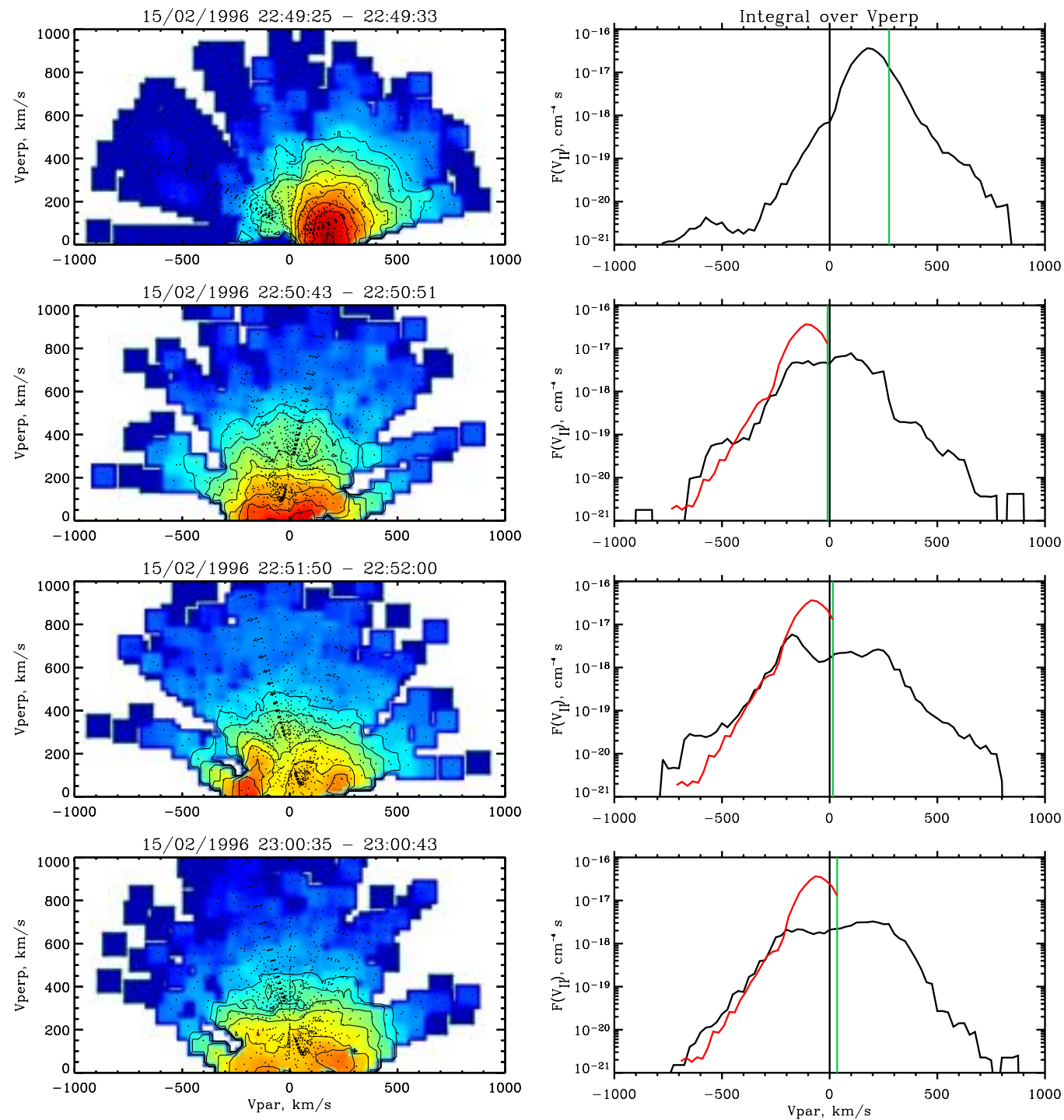

Fig. 11. Magnetosheath velocity distribution is shown as observed before the magnetopause crossing on 15 February 1996 (top). Also shown are three LLBL velocity distributions with high-density, high-speed ion components observed simultaneously, moving along the magnetic field direction and opposite to the magnetic field direction within LLBL interval 3 in Fig. 2. See Fig. 10 for format explanation.

tions for comparison with theoretical expectations. These are the cases when two high density ion components, one moving along the negative magnetic field direction and another one moving along the positive magnetic field direction, were observed simultaneously (cases of two-side injections from the time intervals 3-5 in Fig. 2). Figure 11 shows these cases in comparison with the magnetosheath velocity distribution observed just before the magnetopause crossing. Although there is some similarity between Figs. 10 and 11, there is an important distinction between the two sets of data, most notably seen on the $V_{\|}-V_{\perp}$ color diagrams. The ion component moving along the negative $B$ direction strongly dominates in the distributions shown in Fig. 10, while Fig. 11 shows nearly identical counter-streaming components.

The parallel component of the HT velocity is marked on the magnetosheath velocity distribution of Fig. 11 by a green vertical line. Projections of the same HT velocity to average magnetic field directions for each LLBL $V_{\|}$-velocity distribution are shown by green vertical lines. The red line on each of LLBL spectrum shows a velocity distribution that should be observed if the magnetosheath particles with velocities less than the HT velocity component in the magnetosheath would enter the LLBL field line. This comparison demonstrates that the ion component moving along the negative magnetic field direction in the LLBL has a magnetosheath origin but agreement between the observed and expected locations of maxima of the velocity distributions is not as good as for the onesided injections.

Statistical analysis of two ion components moving in opposite directions within the LLBL regions 3 and 5 on Fig. 2 and in three LLBL transients shown in Fig. 4 was performed. Each component in these velocity distributions was approxi- 


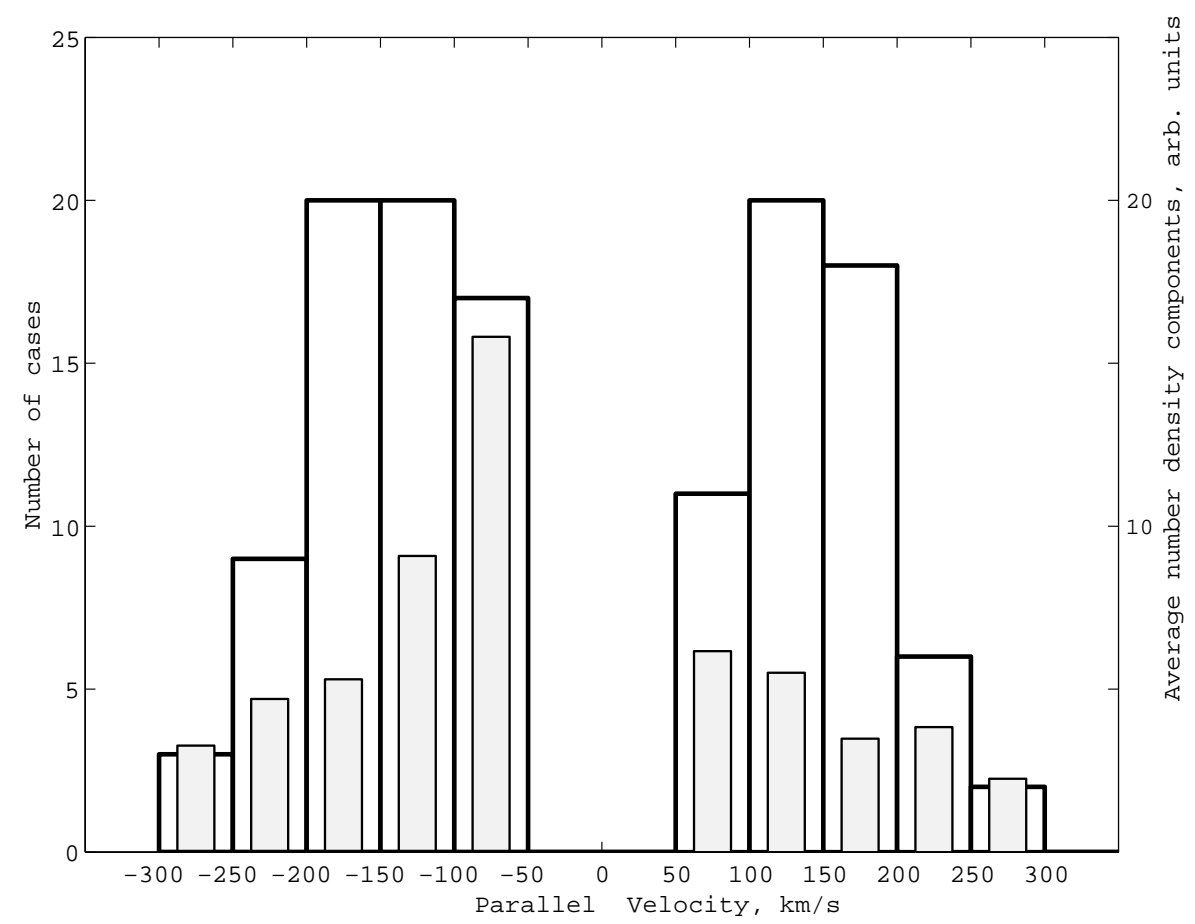

Fig. 12. Statistical distribution of velocities and number densities of counter-streaming components are shown as observed in the LLBL on 15 February 1996. LLBL intervals 3 and 5 from Fig. 2 and three LLBL transients from Fig. 4 are included. Empty columns are convected Maxwellian velocity distributions, gray columns are for average number density of components within a given velocity interval.

mated by the convected Maxwellian velocity distribution (fitted to the upper part of the distribution, within $\sim 1.5$ orders of magnitude of maximum phase space density), in order to obtain the number density, temperature, and convection velocity along the field line. The average temperatures for the ion components are $77 \mathrm{eV}, 105 \mathrm{eV}$, and $73 \mathrm{eV}$ for parallel, central, and anti-parallel velocity components, correspondingly. Maxwellian fits to magnetosheath ion velocity distribution (performed in the same way as for ion components within the LLBL, not by moments' calculations) in the layer close to the magnetopause gives an average temperature of about $65 \mathrm{eV}$. The magnetosheath temperature was higher farther from the magnetopause, but external conditions may vary with time. This analysis confirms that all ion components within the LLBL are of magnetosheath origin. Parallel velocities distributions and number density distributions statistics for LLBL ion components are shown in Fig. 12. Average velocities for anti-parallel components and anti-parallel components are about $140 \mathrm{~km} / \mathrm{s}$. The number density of the parallel component is, on average, two times larger than that for the anti-parallel component.

\subsection{Summary of observations of ion velocity distributions}

Interball-tail observations at the flank of the magnetopause on 15 February 1996 show that ion velocity distributions within the highly structured LLBL are highly variable and often consist of two or three magnetosheath-type components, at positive, negative and nearly zero velocity along the local magnetic field. (a) There are velocity distributions with high-density magnetosheath-like components moving either opposite to the local magnetic field direction or along the magnetic field direction. These components frequently have low-velocity cutoffs (D-shaped velocity distributions), as one would expect to observe on the magnetospheric flux tube reconnected to the magnetosheath magnetic field (Cowley, 1982).

(b) There are cases with two high-density magnetosheathlike components observed simultaneously, one moving opposite to the local magnetic field direction or along the magnetic field direction. These two components may have different velocities, as well as the widths of the spectra. There are the cases when these two counterstreaming components have nearly the same number densities and very similar spectral characteristics.

(c) When one magnetosheath-like component coming from one direction relative to the magnetic field direction dominates, in most cases there is a weak magnetosheath-like component having the opposite velocity relative to the magnetic field direction. Relative number densities of two components seldom exceed 1 order of magnitude.

(d) In many cases the component with zero or nearly zero parallel velocity is a dominant component of ion velocity distribution within LLBL. 


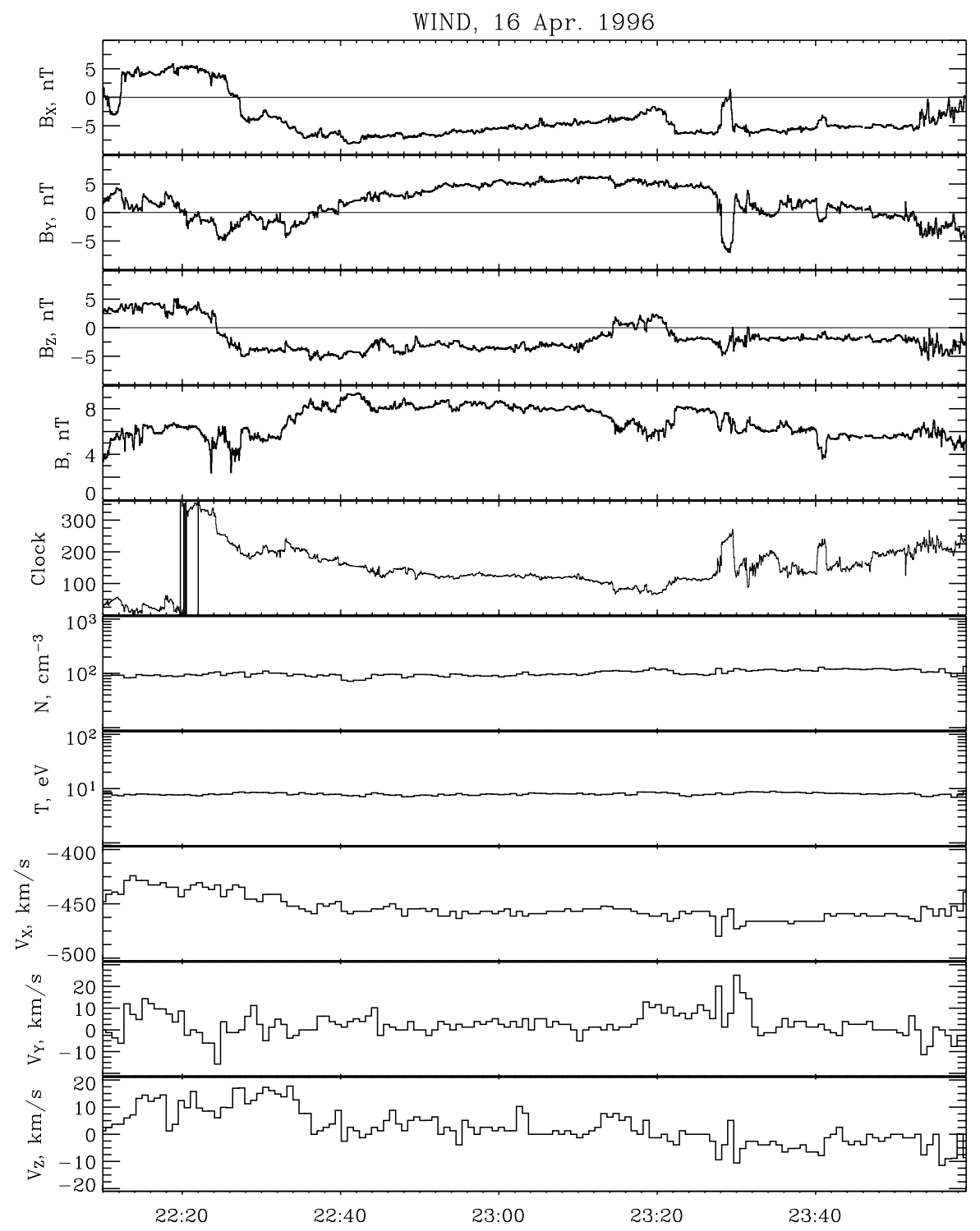

Fig. 13. WIND magnetic field and plasma data for Interball observations of LLBL on 16 April 1996. Format of this figure is the same as for Fig. 1.

(e) As the number density within LLBL parcel decreases, the phase space density of the central $\left(V_{\|} \sim 0\right)$ ion component decreases faster than the phase space density of parallel and anti-parallel velocity components.

\section{Characteristics of the observed LLBL on 16 April 1996}

\subsection{Plasma flow parameters within the LLBL}

The second LLBL crossing was chosen in order to compare the data obtained on 15 February 1996 with the LLBL observed closer to the subsolar point. On 16 April 1996 Interball crossed the magnetopause inbound at 22:42 UT at $X_{\mathrm{GSE}}=6.68 R_{E}, Y_{\mathrm{GSE}}=5.42 R_{E}$, and $Z_{\mathrm{GSE}}=-4.75 R_{E}$,
MLT $=15: 20$, GM latitude $=2.97^{\circ}$. The Wind spacecraft was located close to the Earth at $X_{\mathrm{GSE}}=10.3 R_{E}, Y_{\mathrm{GSE}}=$ $34.1 R_{E}$, and $Z_{\mathrm{GSE}}=-3.4 R_{E}$, providing a small delay in propagation between the Wind and Interball. The solar wind plasma parameters (courtesy of K. Ogilvie) were: ion number density $\sim 12 \mathrm{~cm}^{-3}$, velocity $\sim 450 \mathrm{~km} / \mathrm{s}$, and ram pressure $\sim 4 \mathrm{nPa}$.

The IMF (courtesy of R. Lepping) turned from northward to southward at $\sim 22: 24$ UT, shortly before Interball crossed the magnetopause and remained southward, except for 23:14:30-23:21 UT when it changes the sign several times (Fig. 13). Most of the time interval when Interball observed plasma transients after the magnetopause crossing, the clock angle of the IMF was in the range of $110^{\circ}-$ $120^{\circ}$. The Geotail spacecraft was located in the magne- 


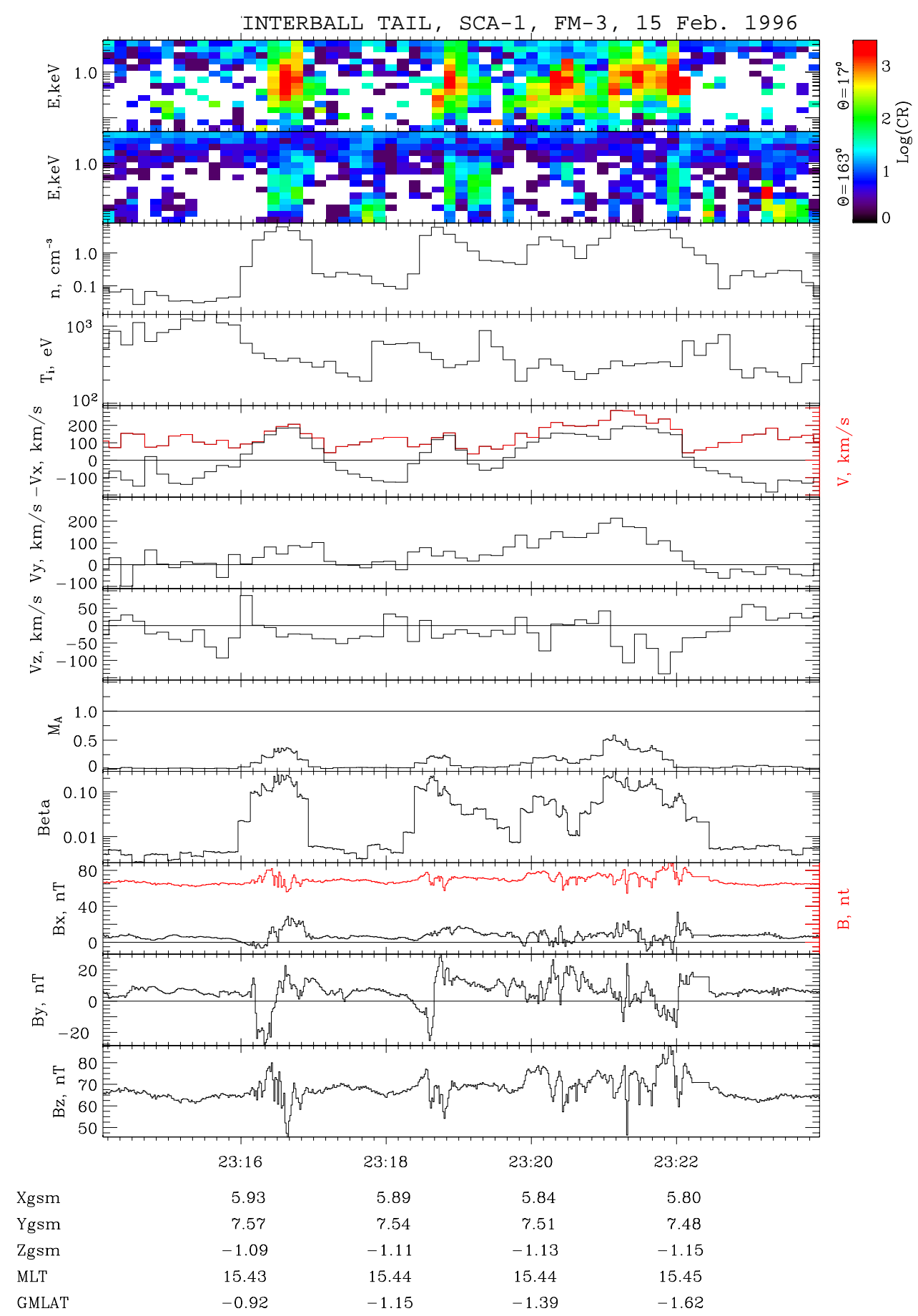

Fig. 14. Transients observed on inbound trajectory of Interball on 16 April 1996. Format is the same as in Fig. 2.

tosheath on the magnetospheric flank at $X_{\mathrm{GSE}}=-6.20 R_{E}$, $Y_{\mathrm{GSE}}=26.60 R_{E}$, and $Z_{\mathrm{GSE}}=-2.50 R_{E}$, also at a small delay with Interball. Geotail observed the southward magnetic field for the time interval under discussion.

At $\sim 50 \mathrm{~min}$ after the magnetopause crossing, Interball observed many short-duration $(0.5-1 \mathrm{~min})$ bursts of magnetosheath-like plasma (Fig. 14). This type of highly structured LLBL is usually observed under southward or variable IMF conditions (Vaisberg et al., 2001). There are variations of magnetic fields associated with these events, but only the first one shows a typical FTE signature when the magnetic field is converted to the normal coordinate frame (not shown). For a given IMF clock angle we should also expect that the reconnection site will be located in the northdusk sector of the dayside magnetopause, like we discussed for 15 February 1996 case. Values of number density and 

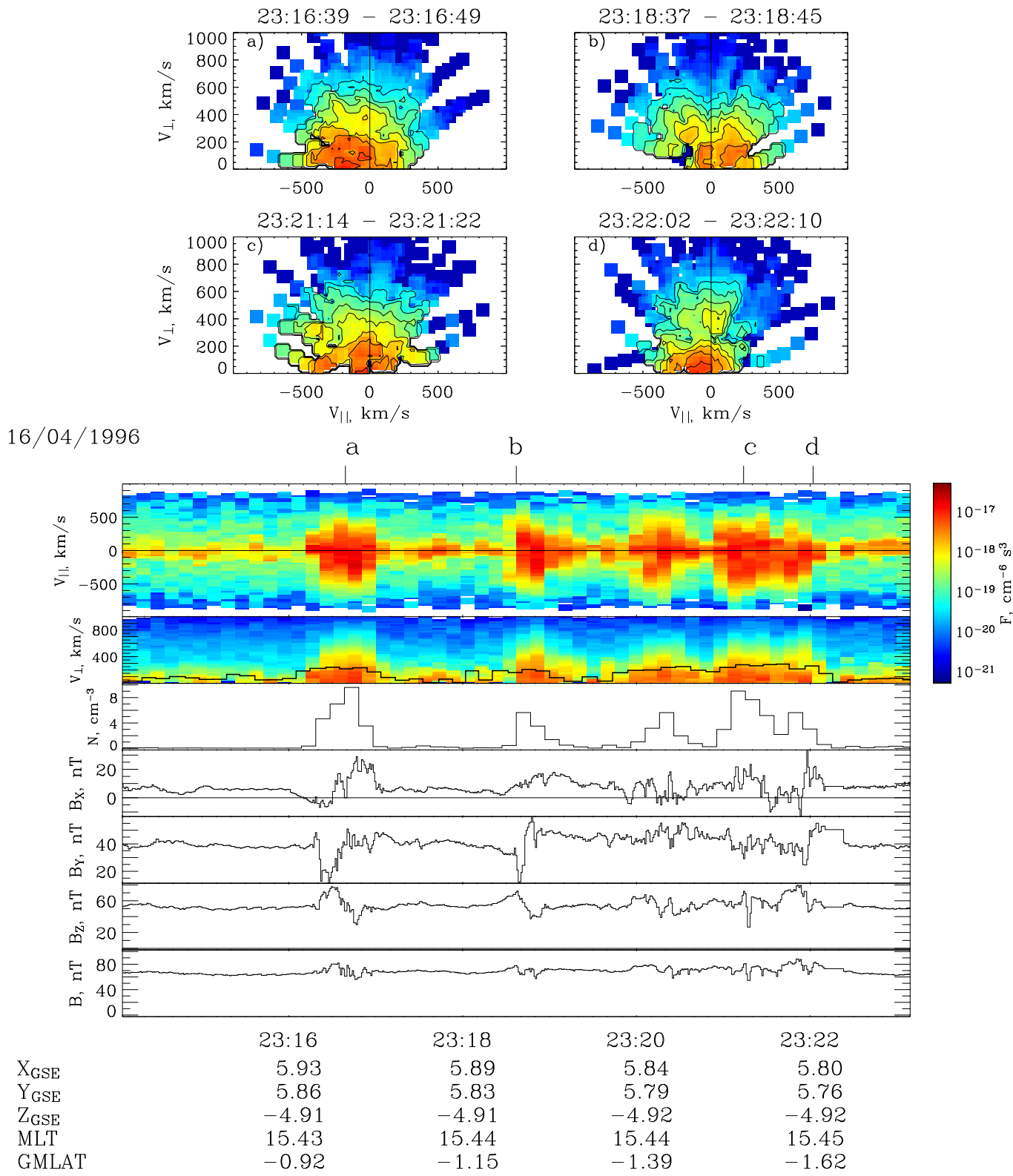

Fig. 15. Four representative ion velocity distributions within a set of transients observed within the LLBL on 16 April 1996 (top). $V_{\|}$-time and $V_{\perp}$-time spectrograms, number density and magnetic field data are shown on the bottom. Times for velocity distributions are shown by tick marks. See Fig. 10 caption for more explanation.

temperature within these plasma transients are different from those in the magnetosheath. This indicates that these plasma transients are not the multiple magnetopause crossings rather than LLBL part.

\subsection{Ion velocity distributions within the LLBL observed on 16 April 1996}

Figure 15 shows the representative ion velocity distributions observed within four transients shown in Fig. 14. The times when these velocity distributions were observed are shown by tick-marks above the $V_{\|}-V_{\perp}$ velocities-time spectrograms. The $V_{\|}$-velocity-time spectrogram shows that sig- nificant ion flux both parallel to the magnetic field direction and anti-parallel to the magnetic field direction is observed most of the time. The flux antiparallel to the magnetic field direction dominates. There are several types of $V_{\|}-V_{\perp}$ spectra within these four transients: (a) a strong flux opposite to the magnetic field direction with a central component and a weak component in the positive $B$ direction, (b) nearly identical components in two directions relative to the magnetic field, (c) a fast component in the negative $B$ direction, an intense component at low parallel velocities, and some flux in the positive $B$ direction, and (d) a dominant component at $V \sim 0 \mathrm{~km} / \mathrm{s}$ with weak flux in two directions. 


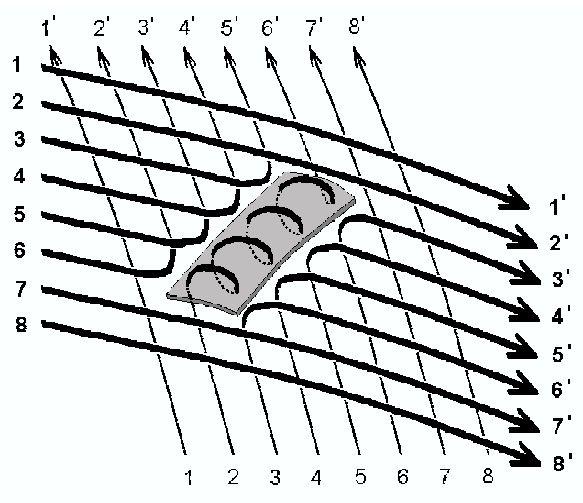

A

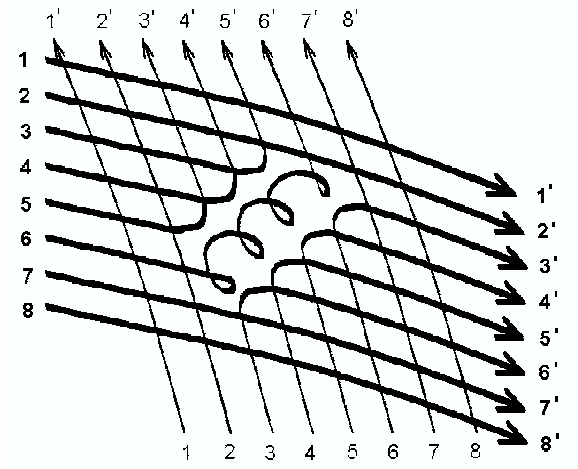

C

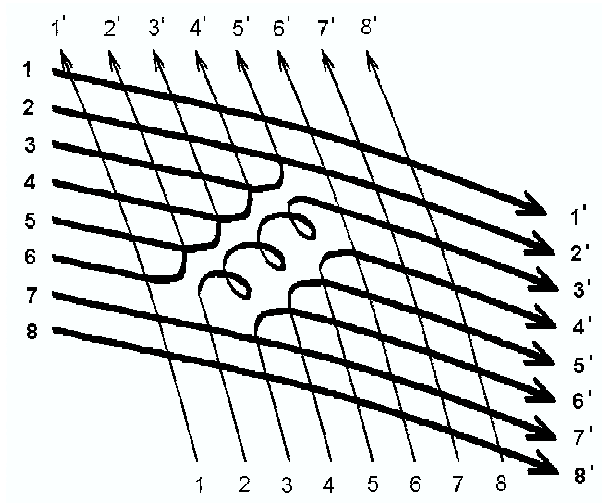

B

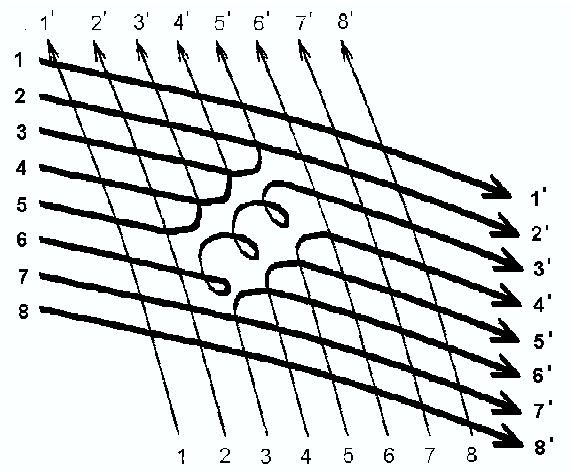

D

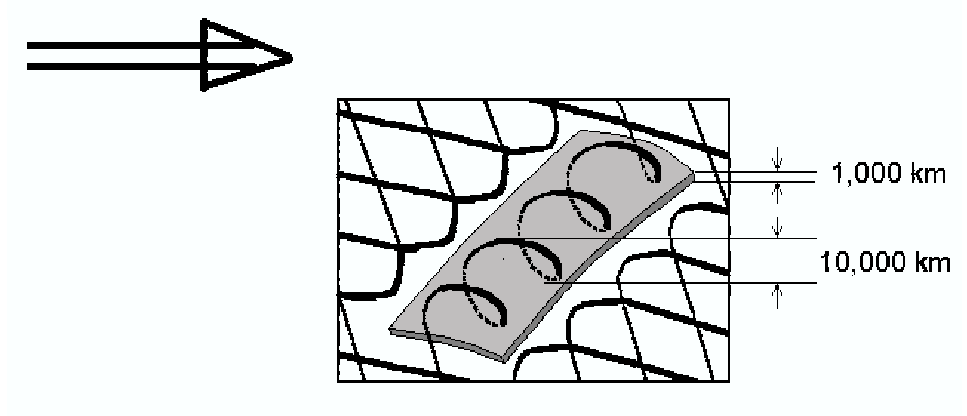

$\mathbf{E}$

Fig. 16. Flux rope diagram partly adopted from computer simulations results (Lee et al., 1993). Thick lines indicate magnetosheath magnetic field lines that are located closer to the viewer, while thin lines indicate background magnetospheric field lines. Field lines are numbered to make viewing easier. Four cases are shown: both ends of the flux rope connected to the magnetosphere (A), one end connected to the magnetosheath and another end connected to the magnetosphere $(\mathbf{B})$ and $(\mathbf{C})$, and both ends connected to the magnetosheath (D). The spatial relationship of magnetic field lines is most readily seen in drawing A. Thick lines show magnetic field line segments loaded with magnetosheath plasma, thin segments are loaded with magnetospheric plasma. Large arrow shows magnetosheath flow direction. Directions of magnetic fields and magnetosheath flow are close to conditions observed on 15 February 1996 and on 16 April 1996. Inset on the bottom shows how the flux rope relates to the magnetospheric current sheet and indicates their linear scales.

In summary, Interball-Tail observations at dayside magnetopause on 16 April 1996 show similar types (comparing to 15 February 1996) of ion velocity distributions within the LLBL structures, with two or three magnetosheath-type components, at positive, negative and nearly zero velocity along the local magnetic field. Thus, measurements within the dayside LLBL transients on 16 April 1996 confirm the conclusions drawn from the 15 February 1996 LLBL observations. Two events frequently show multi-component ion velocity distributions within the LLBL. 


\section{Discussion}

\subsection{Counter-streaming components}

Reconnection at a single $X$-line produces open magnetospheric flux tubes that connect to magnetosheath flux tubes through a rotational discontinuity. Parts of the magnetospheric and magnetosheath plasma velocity distributions cross the open magnetopause freely and form D-shaped velocity distributions (Cowley, 1982). This type of ion velocity distributions has been observed previously (e.g. Smith and Rodgers, 1991; Phan et al., 2001), and Interball measurements also provide examples of this type of D-shaped distributions at the edges of LLBL features and sometimes within them.

However, in addition to the observed one-sided injection of the magnetosheath plasma along the field line, the Interball spacecraft also observed counter-streaming injections in the LLBL and, additionally, the third component at $\sim$ zero parallel velocity in local magnetic coordinate frame. The ion component coming from a direction opposite to the ion component entering the magnetosphere from the magnetosheath along the open field line can be produced by reflection of earlier injected ions from parts of the same field line closer to the Earth, where the magnetic field strength is much higher (this is frequently called reflection from the ionosphere). The possibility of obsserving the ions reflected from the ionosphere can be estimated by comparison of convection time of the LLBL flux tube along the magnetopause and the propagation time of ions along the field line. Typical parallel velocity of ions in the LLBL is $\sim 150 \mathrm{~km} / \mathrm{s}$. Two-way travel time along the field line of $\sim 15 R_{E}$ for these ions will be $\sim 10-20 \mathrm{~min}$, depending on the pitch-angle. In the case where reconnection occurs close to the subsolar point, the LLBL plasma with bulk velocity $\sim 200 \mathrm{~km} / \mathrm{s}$ will convect to the flank of the magnetosphere ( 15 February 1996 case) within $\sim 15 \mathrm{~min}$, comparable or faster than the reflection time. For dayside LLBL (16 April 1996 case) the propagation time along the field line is much longer than convection along the magnetopause. Ionospheric reflection is strongly pitch-angle dependent, as well as energy dependent, and will result in a velocity distribution with limited energy spread and with higher energies observed at smaller pitch angles. In two cases of LLBL crossings we discuss in this paper, such dispersed velocity distributions in the ion component moving along the positive direction of the magnetic field have not been observed. We often see symmetric velocity distributions for the counter-streaming ions in the LLBL, both at the flank magnetopause and on the dayside. No deficit of low-velocity plasma that should be seen in ions reflected from the ionosphere is observed in the dayside LLBL. Frequently, the plasma coming from "ionospheric" direction is actually denser than the one coming from "direct" entry. This is difficult to expect for plasma that had to propagate for $\sim 10-20 \mathrm{~min}$ along the field line and to experience significant dilution due to drifts and atmospheric losses. This makes the explanation of oppositely directed ions within the LLBL quite unlikely. The similarity of spectral properties of plasma beams in the LLBL coming from opposite directions suggests their similar origin.

The existence of the two ion components moving opposite to each other is quite a common feature in the LLBL, as observed on the two days. There are cases of a high-density D-shaped component, indicating magnetosheath plasma injection along open field lines, more frequently along the negative direction of the magnetic field corresponding to a reconnection site northward of the spacecraft. In these cases a lower density component moving in the opposite direction is also observed. There are also cases of two co-existing highdensity ion components moving in opposite directions along the magnetic field.

Simultaneous observations of counter-streaming magnetosheath-type components indicate that the field line is open for entry of the magnetosheath plasma on two sides. This suggests multiple reconnections but does not require that these reconnections occurr exactly simultaneously. These observations suggest that multiple reconnections in which magnetosheath and magnetospheric field lines reconnect at more than one location plays a certain role in the dayside of the magnetosphere. Multiple reconnections allow for the magnetosheath plasma to enter the magnetospheric field lines from two directions and to be observed at one location as counterstreaming components.

Figure 16 is a simplified scheme of flux ropes that may form at multiple reconnection of magnetospheric and magnetic fields between two $X$-lines for the magnetospheric and magnetosheath magnetic field directions close to those observed on 15 February 1996, and on 16 April 1996. These are views of the magnetopause from outside. Magnetosheath field lines (shown as thick lines) are closer to the viewer as a magnetospheric field lines (shown as thin lines) are farther from the viewer. An arrow indicates the direction of the magnetosheath flow. Four cases shown in Fig. 16 correspond to different cases of magnetic connection to magnetospheric or magnetosheath field lines. Due to multiple reconnections "the ends" of the spiral may be connected to different domains (cases B and C) or to the same domain (cases A and D). In the latter cases "the ends" connect to originally different field lines in the same domain. To more clearly understand the third dimension in the two-dimensional picture, magnetospheric field lines in case A are shown by dashes between magnetosheath field lines. This emphasizes that magnetospheric field lines are below (farther from the viewer) magnetosheath field lines. The location of the magnetopause current layer before the flux rope was formed is also schematically shown on the scheme A by a gray sheet.

The size of a reconnected flux tube or FTE (Elphic, 1995) is significantly larger than the thickness of the magnetopause current layer (Lee and Russell, 1994). Accordingly, some sectors of magnetic spiral are loaded with magnetosheath plasma (thick sectors of the spiral), while another part of the spirals is filled with magnetospheric plasma (thin sectors). The inset $\mathrm{E}$ on the bottom of Fig. 16 schematically shows the relation of the flux rope to the magnetopause current layer and their approximate scales. Simulation also indicates that 

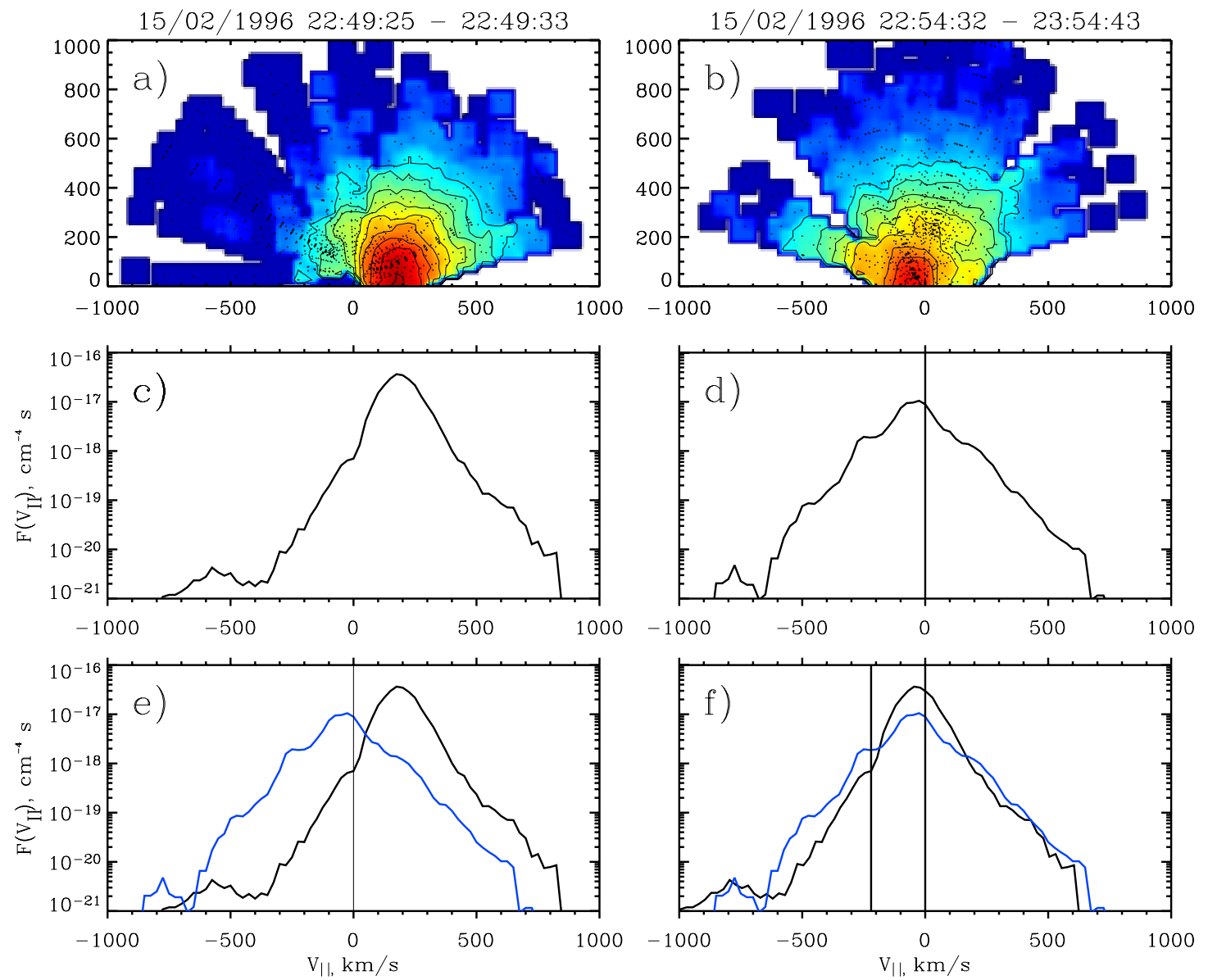

Fig. 17. A comparison is given of magnetosheath ion velocity distribution (panel (a) and panel (c)) with typical 3-component distribution in the LLBL (panel (b) and panel (d)). Upper diagrams are cuts through velocity distributions in the magnetic coordinate system, next row: the same distributions integrated over $V_{\perp}$. Panel (e) shows spectra (c) and (d) superimposed without displacement along $V_{\|}$. Panel (f) is comparison of the LLBL spectrum (d) with magnetosheath spectrum (c) displaced to negative $V_{\|}$velocities for co-location spectra maximums. Vertical line indicates the amount of displacement of spectrum (c) along $V_{\|}$.

the flux rope formed by multiple reconnections consists of two parts, one of which comes from the magnetosheath, and one comes from the magnetosphere (Lee et al., 1993).

The sectors of the flux tube loaded with the magnetosheath plasma are the sources of the magnetosheath-type plasma to the sectors with a magnetospheric population. Particles having large parallel velocity will enter the sectors with magnetospheric population and form a bi-directional magnetosheath-type velocity distribution. The evolution of the ion velocity distribution within the sectors of the flux rope with original magnetosheath population is discussed in the next paragraph.

As it was mentioned in Sect. 3.2, the most probable location of the reconnection site is the north-dusk sector. The observations were made in the region of super-Alfvénic magnetosheath flow where reconnection is improbable. The geometry of field lines should undergo a significant evolution during the convection time, and the spiral structure shown in Fig. 16 will evolve due to magnetic diffusion, since the turbulence would provide a sufficient resistivity. This will lead to the evolution of the velocity distribution, as well. However, the simplified scheme shown in Fig. 16 allows us to explain some features of observed velocity distributions.

\subsection{Component with nearly zero parallel velocity}

The ions entering from the magnetosheath to the magnetosphere along open magnetic flux tubes form asymmetric D-shaped ion velocity distributions (Cowley, 1982), rather than a component centered at zero parallel velocity. Maximum phase space density at zero parallel velocity would be observed for reconnection close to the subsolar point where magnetosheath plasma velocity is low. However, the most probable reconnection site for our cases is the northdusk sector (see Sect. 3.2), where the magnetosheath velocity component along the magnetosheath magnetic field direction should be large for our conditions. The ion velocity distributions observed in the LLBL have a component with a maximum at nearly zero parallel velocity and is approximately at rest relative to the magnetic field. Ionospheric ions 
are one of the possibilities to explain this component. Indeed, as one may see in Fig. 15, the ionospheric component is easily seen at $V_{\|} \sim 0 \mathrm{~km} / \mathrm{s}$ before, and especially after four transients were observed. Paschmann et al. (1986) and Klumpar et al. (1990) reported the existence of ionospheric ions in the LLBL. However, as observed by Interball, on 15 February 1996 and on 16 April 1996, the ion component at small parallel velocity in the LLBL usually had a temperature of the same order of magnitude as the temperature of the magnetosheath plasma, that does not agree with the ionospheric source.

Figure 17 shows the comparison of the ion velocity distribution observed by Interball on 15 February 1996 in the magnetosheath just before the magnetopause crossing, with the typical velocity distribution in the transient (see times above $V_{\|}$-ion velocity distributions). The phase space density at $V_{\|} \sim 0 \mathrm{~km} / \mathrm{s}$ in the magnetosheath is smaller than in the LLBL by almost one order of magnitude (Fig. 17e) which makes it difficult to explain this LLBL component at $V_{\|} \sim 0$ by magnetosheath origin. This component could enter the LLBL closer to the subsolar point, where the velocity of the magnetosheath plasma is smaller, and the velocity cutoff for transmitted ions is smaller, as well. However, this component at $V_{\|} \sim 0$ is observed in the LLBL simultaneously with newly-injected plasma. It means that the injection of the magnetosheath plasma into the LLBL could happen twice, once closer to the subsolar point, and a second time at the location closer to the flank of the magnetosphere. This is not an improbable scenario.

Another possibility to explain the frequent coexistence of a component with nearly zero parallel velocity, with two components of ions moving along and opposite to the magnetic field direction, lies in the multiple reconnection scenario. Magnetosheath plasma approaching the magnetopause is initially located on the magnetic flux tubes that do not cross the magnetopause. When a particular magnetosheath flux tube reconnects with a magnetospheric one, the current is injected in this flux tube that forms the flux rope. The change in the magnetic field direction leads to the change in the angle between the plasma flow vector and the magnetic field vector. The direction of the magnetic field within the flux rope is intermediate between the directions of the magnetosheath magnetic field and the direction of the magnetospheric magnetic field (Fig. 16). Simulations of multiple reconnection show that plasma filling a newly-formed flux rope has a significant bulk velocity directed away from reconnection location (Fu et al., 1990). Since the magnetic field within the flux rope has a spiral structure, there are sectors within this flux rope where the bulk velocity has a small component along the magnetic field direction (see Fig. 2 in Fu et al., 1990). This leads to an increase in the phase space density at small parallel velocity and may explain the origin of the central component in the velocity distribution.

Some properties of ion velocity distribution support the supposition of this transformation of ion velocity distribution in the magnetic coordinate system. The first property is in very similar shapes and symmetries to the two velocity distributions (parallel and anti-parallel) at $\left|V_{\|}\right|<\sim 150 \mathrm{~km} / \mathrm{s}$ (Fig. 17). The second one is the high-energy component seen at $V_{\|} \sim-600 \mathrm{~km} / \mathrm{s}$ within the magnetosheath and at $V_{\|} \sim-800 \mathrm{~km} / \mathrm{s}$ within the LLBL. If we shift the magnetosheath velocity distribution along the magnetic field direction to make its maximum nearly coincide with the maximum of the LLBL velocity distribution, these high-energy components also quite closely coincide. The third detail of the two velocity distributions is their velocity cutoff at high positive $V_{\|}$that becomes nearly matching after the shift in the magnetosheath velocity distribution.

Figure 17 also shows that the excess of the magnetosheathtype ions at higher parallel velocities is quite a common property of the LLBL ion spectra. The phase space density in the LLBL velocity distribution at velocities $\left|V_{\|}\right|>\sim 200 \mathrm{~km} / \mathrm{s}$ is in excess in comparison with the shifted magnetosheath velocity distribution. It was mentioned earlier that for LLBL parts with a lower number density the relative contribution of the central component decreases compared to two counterstreaming components. This change in the velocity distribution with ion number density results in the inverse relationship between $N$ and $T$ in the LLBL.

These observations suggest that multiple reconnections in which magnetosheath and magnetospheric field lines reconnect at more than one location is taking place on the dayside magnetosphere. Formation of the flux rope at the magnetopause will lead to the development of two-component and three-component velocity distributions. The ions from the wings of the velocity distribution in the parts of the flux rope that were filled originally with magnetosheath plasma stream along field lines into the regions that are filled by magnetospheric plasma. In this part of the flux rope they form the bimodal ion distribution of magnetosheath-type particles. The ions with small parallel velocities remain at their initial locations. Ions with high parallel velocities that escaped from their original locations along field lines to former magnetospheric parts, will return back to the magnetosheath parts of the rope and form the wings of the ion distribution (Fig. 9).

Interball observations show that the process leading to the decrease in the number density in the LLBL is accompanied by a faster decrease in the phase space density in the central component of the velocity distribution compared to the wings. This indicates that more ions leave the parts of the flux rope that came from the magnetosheath and contribute to the wings of the velocity distribution. Therefore, the concept of multiple reconnections is potentially able to explain important properties of the ion velocity distribution within the LLBL and its evolution with decreasing number density (that appears to be the result of the "aging" of the LLBL plasma). Two-component and three-component ion velocity distributions within the LLBL suggest that the flux ropes at the magnetopause can be formed by multiple reconnections.

As was mentioned earlier, convection of the spiral from the reconnection site to the observation point will modify the shape of these spiral and velocity distributions contained within it. However, observations indicate that ion velocity distributions within the LLBL frequently consist of two or 
three components of the magnetosheath type. This indicates their origin and shows that the single-component hot distribution is not commonly produced in the LLBL, either as a result of reconnection or due to full relaxation of two- and three-component distribution.

\subsection{Implication to magnetic topology}

There are a number of properties of ion velocity distributions within the LLBL that indicate that a significant part of the LLBL consists of flux ropes that have a quasi-closed magnetic topology.

- A significant part of the LLBL transients has a convection velocity close to the one observed in the nearby magnetosheath while moving at the large angle to the magnetospheric magnetic field direction, with kinetic energy of plasma exceeding magnetic energy density.

- There are cases with two high-density magnetosheathtype ion components moving parallel and anti-parallel to the local magnetic field direction, indicating fresh injections of magnetosheath plasma from two locations.

- There are cases within the LLBL when velocity distributions do not show typical D-shaped components that should be observed at the magnetospheric magnetic field line reconnected with the magnetosheath magnetic field.

- There are cases when the component at nearly zero parallel velocity is a dominant component in the velocity distribution. Its phase space density may exceed the one within the magnetosheath velocity distribution at the same parallel velocity.

- There is redistribution of relative density between the ion component at nearly zero parallel velocity and two ion components moving opposite to and along the local magnetic field.

- The average magnetic field within the LLBL has an intermediate orientation between the directions of magnetosheath and the magnetospheric field lines.

These LLBL properties observed in two LLBL crossings can be explained in the concept of the flux rope formed as a result of multiple reconnections. This flux rope has some sectors loaded with magnetosheath plasma, while other sectors are loaded with magnetospheric plasma. Only part of the flux rope is directly magnetically connected to the magnetosheath or magnetospheric field lines. Depending on the location of the spacecraft within this complicated structure it can observe the injection of magnetosheath plasma from one or another direction along the field line, or both, or observe the higher-density component at nearly zero parallel velocity (central component) with two counter-streaming magnetosheath-type components. The escape of particles with small parallel velocity from the central component will feed two counter-streaming components.
Since a significant part of the flux rope formed as the result of multiple reconnections is filled with high-density, high-velocity magnetosheath plasma, it will propagate approximately along the magnetosheath flow, and its kinetic energy may exceed the magnetic field energy. Some parts of the LLBL may not be considered as regular magnetospheric boundary layer regions. These flux ropes propagate between the magnetosheath flow and the magnetosphere, and may not be topologically connected with any of these regions.

\section{Conclusion}

Interball measurements for two highly-structured LLBL crossings under southward and variable magnetic field magnetosheath conditions show that ion velocity distributions within the LLBL can be separated into several categories, the primary of which are: (a) one component moving along (or opposite to) the local magnetic field, (b) two components moving in opposite directions relative to the magnetic field, and (c) three components, one of which has nearly zero velocity along the magnetic field and two components moving in opposite directions relative to the magnetic field. Category (a) is observed mainly at the edges of LLBL structures and is the typical D-shape distribution observed in reconnected magnetospheric field lines (Cowley, 1982). Category (b) is observed at the edges of LLBL structures as well as within these structures. Two oppositely moving ion components usually have similar spectral characteristics but their relative densities vary. Their similar spectral characteristics, absence of velocity/pitch-angle separation for a component that may be considered as one reflected from the ionosphere, and the long return flight time to the ionosphere and back compared to the LLBL plasma convection time, rule out the possibility of explaining the second component by ionospheric reflection. These two oppositely moving components can be explained by the reconnection of particular magnetic flux tubes in two sites, or by the propagation of ions along closed field lines from the higher density region. Category (c) is observed within LLBL structures; the existence of a component that is at rest in the magnetic coordinates is not compatible with reconnection of the single magnetospheric flux tube at one location, as described in (Cowley, 1982). None of its characteristics could be explained by the ionospheric component. This ion component could be explained by the trapping of magnetosheath plasma within the flux rope forming at the magnetopause by multiple reconnections.

Category (c) ion spectra behave in the systematic way within the LLBL. Going from higher density parts of the LLBL to lower density parts we see that the relative contribution of the velocity distribution component that is at rest in the magnetic coordinates is diminished, and the relative contribution of the wings of the velocity distribution, that represent components moving along the field line in opposite directions. This could be explained by the redistribution of ions along "closed" field lines from higher density parts of the flux rope formed by multiple reconnections. 
In summary, we have observed the ion characteristics of ions within the LLBL that cannot be explained by the reconnection of the magnetosheath and the magnetospheric field lines at one location. We argue that reconnection at two or more locations, leading to the formation of the flux ropes at the magnetopause, can explain observed velocity distributions. We consider these observations as evidence for multiple reconnections between magnetosheath and magnetospheric flux tubes with the formation of spiral magnetic flux tubes (Lee and Fu, 1985). This implies that the multiple reconnections are at times an important factor in the formation of the LLBL.

Acknowledgements. Authors gratefully acknowledge very useful discussions with A. Otto, V. Osherovich, A. Kropotkin, L. Zelenyi, and A. Petrukovich.

Topical Editor T. Pulkkinen thanks M. Hapgood and another referee for their help in evaluating this paper.

\section{References}

Cowley, S. W. H.: The causes of convection in the Earth's magnetosphere: A review of developments during the IMS, Rev. Geophys., 20, 531, 1982

Crooker, N. U.: Dayside merging and cusp geometry, J. Geophys. Res., 84, 951-959, 1979.

De Keyser J., Darrouzet, F., Roth, M., Vaisberg, O. L., Rybjeva, N., Smirnov, V., Avanov, L., Nemecek, Z., and Safrankova, J.: Transients at the dawn and dusk side magnetospheric boundary: Surface waves or isolated plasma blobs? J. Geophys. Res., 106, 25 503-25 516, 2001.

Dungey, J. W.: Interplanetary magnetic field and the auroral zones, Phys. Rev. Lett., 6, 47, 1961.

Eastman, T. E., Hones, Jr., E. W., Bame, S. J., and Asbridge, J. R.: Magnetospheric boundary layer: Site of plasma, momentum, and energy transfer from magnetosheath into magnetosphere, Geophys. Res. Lett., 3, 685-688, 1976.

Elphic, R. C.: Observations of Flux Transfer Events: A review, in: Physics of the Magnetopause, Geophys. Monograph 90, edited by Song, P., Sonnerup, B. U. O., and Thomsen, M. F., AGU, pp. 225-233, 1995.

Fu, Z. F., Lee, L. C., and Shi, Y.: A three-dimensional MHD simulation of the multiple $X$-line reconnection process, in: Physics of Magnetic Flux Ropes, Geophys. Monogr. Ser., vol. 58, edited by Russell, C. T., Priest, E. R., and Lee, L. C., pp. 515-519, AGU, Washington, D.C., 1990.

Hapgood, M. A., and Bryant, D. A.: Re-ordered electron data in the low latitude boundary layer, Geophys. Res. Lett., 17, 2043, 1990.

Hau L.-N. and Sonnerup, B. U. O.: Two-dimensional coherent structures in the magnetopause: Recovery of static equilibria from single-spacecraft data, J. Geophys. Res., 104, 6899, 1999.

Khrabrov, A. V. and Sonnerup, B. U. O.: DeHoffman-Teller analysis, in: Analysis Methods for Multi-spacecraft Data, edited by Paschmann, G. and Daly, P. W., ISSI Sci. Rep. SR-001, p. 221248, ESA Publication Division, Noordwijk, The Netherlands, 1998.

Klimov, S., Romanov, S., Amata, E., Blecki, J., et al.: ASPI experiment. Measurements of fields and waves onboard the Interball-
Tail mission, in Interball mission and payload, RSA-IKI-CNES, 120-152, 1995.

Klumpar, D. M., Fuselier, S. A., and Shelley, E. G.: Ion composition measurements within magnetospheric flux transfer events. Geophys. Res. Lett., 16, 2305, 1990.

Lee, G. and Russell, C. T.: The thickness and structure of high beta magnetopause current layer, Geophys. Res. Lett., 21, 2451, 1994.

Lee, L. C. and Fu, Z. F.: A theory of magnetic flux transfer at the Earth's magnetopause, Geophys. Res. Lett., 12, 105, 1985.

Lee, L. C., Ma, Z. W., Fu, Z. F., and Otto, A.: Topology of magnetic flux ropes and formation of fossil flux transfer events and boundary layer plasma, J. Geophys. Res., 98, 3943, 1993.

Lockwood, M. and Hapgood, M. A.: On the cause of a magnetospheric flux transfer event, J. Geophys. Res., 103, 26 453-26 478, 1998.

Luhmann, J. G., Walker, R. J., Russell, C. T., Crooker, N. U., Spreiter, J. G., and Stahara, S. S.: Patterns of potential magnetic field merging sites on the dayside magnetopause, J. Geophys. Res. 89, 1739-1742, 1984.

Nishida A.: Can random reconnection on the magnetopause produce the low latitude boundary layer, Geophys. Res. Lett., 16, 227, 1989.

Mooere, T. T., Fok, M.-C., and Chandler, M. O.: The dayside reconnection X line, J. Geophys. Res., 107 (A10), 10.1029/2002JA009381, p. SMP 26, 2002.

Paschmann, G., Papamastorakis, I., Baumjohan, W., Schopke, N., Carlson, C. W., Sonnerup, B. U. O., and Lühr, H.: The magnetopause for large magnetic shear: AMPTE/IRM observations, J. Geophys. Res., 91, 11 099-11 115, 1986.

Petcheck, H. E.: Magnetic field annihilation, AAS-NASA Symposium of Physics of Solar Flares, NASA SP50, 245, 1964.

Phan T. D., Sonnerup, B. U. O., and Lin, R. P.: Fluid and kinetic signatures of reconnection at dawn tail magnetopause: Wind observations, J. Geophys. Res., 106, 25 489, 2001.

Russell C. T. and Elphic, R. C.: Initial ISEE magnetometer results: Magnetopause observations, Space Sci. Rev., 22, 691, 1978.

Sauvaud, J. A., Barthe, H., Aoustin, C., Tocaven, J. J., Rouzaud, J., Bouyssou, J., Cassignol, M., Souleille, P., Bosqued, J. M., Beutier, T., Crasnier, J., Dandouras, J., Jacquey, C., Rème, H., Vaisberg, O., Borodkova, N., and Avanov, L.: The ELECTRON spectrometer experiment: a top hat spectrometer for the Tail Probe, in: Interball Mission and Payload, RKA-IKI-CNES, pp. 153169, 1995.

Sckopke, N., Paschmann, G., Haerendel, G., Sonnerup, B. U. O., Bame, S. J., Forbes, T. G., Jones, E. W., and Russell, C. T.: Structure of the low boundary layer, J. Geophys. Res., 86, 2099, 1981.

Sibeck, D. G. and Smith, M. F.: Magnetospheric plasma flows associated with boundary waves and flux transfer events, Geophys. Res. Lett., 19, 1903-1908, 1992.

Sibeck, D. G., Prech, L., Safrankova, J., and Nemecek, Z.: Twopoint measurements of the magnetopause: Interball observations, J. Geophys. Res., 105, 237-244, 2000.

Smith, M. F. and Rodgers, D. J.: Ion distributions at the dayside magnetopause, J. Geophys. Res., 96, 11 617, 1991.

Sonnerup, B. U. O., Paschmann, G., Papamastorakis, I., Schopke, N., Haerendel, G., Bame, S. J., Asbridge, J. R., Gosling, J. T., and Russell, C. T.: Evidence for magnetic field reconnection at the earth's magnetopause, J. Geophys. Res., 86, 10 049-10 067, 1981.

Sonnerup, B. U. O., Papamastorakis, I., Paschmann, G., and Lühr, H.: Magnetopause properties from AMPTE/IRM observations of convective electric field: Method development, J. Geophys. Res., 
92, 12 137-12 159, 1987.

Thomsen M. F., Stansberry, J. A., Bame, S. J., Fusilier, S. A., and Gosling, J. T.: Ion and electron velocity distributions within flux transfer events., J. Geophys. Res., 92, A11, 12 127-12 136, 1987. Vaisberg, O. L., Leibov, A. W., Avanov, L. A., Smirnov, V. N., Ivanova, E. B., Klimashev, A. A., Khazanov, B. I., Cherkashin, I. I., Iovlev, M. V., Safronov, A. Yu., and Lichtenstein, V. H.: Complex plasma analyzer SCA-1, in Interball Mission and Payload, RKA-IKI-CNES, 170-177, 1995.
Vaisberg, O. L., Smirnov, V. N., Avanov, L. A., Waite, Jr., J. H., Burch, J. L., Russell, C. T., Skalsky, A. A., and Dempsey, D. L.: Observation of isolated structures in the low latitude boundary layer with Interball Tail Probe. Geophys. Res. Lett., 25, 4305, 1998.

Vaisberg, O. L., Smirnov, V., Avanov, L., Waite, Jr., J. H., Burch, J. L., Gallagher, D. L., and Borodkova, N. L.: Different types of low-latitude boundary layer as observed by Interball Tail probe, J. Geophys. Res., 106, 13 068, 2001. 